\title{
Identification of cholesterol-assimilating actinomycetes strain and application of statistical modeling approaches for improvement of cholesterol oxidase production by Streptomyces anulatus strain NEAE-94
}

Noura El-Ahmady El-Naggar ${ }^{*}$ and Nancy M. El-Shweihy

\begin{abstract}
Background: Cholesterol oxidase biosensors have been used to determine the level of cholesterol in different serum and food samples. Due to a wide range of industrial and clinical applications of microbial cholesterol oxidase, isolation and identification of a new microbial source $(s)$ of cholesterol oxidase are very important.

Results: The local isolate Streptomyces sp. strain NEAE-94 is a promising source of cholesterol oxidase. It was identified based on cultural, morphological and physiological characteristics; in addition to the 16S rRNA sequence. The sequencing product had been deposited in the GenBank database under the accession number KC354803. Cholesterol oxidase production by Streptomyces anulatus strain NEAE-94 in shake flasks was optimized using surface response methodology. The different process parameters were first screened using a Plackett-Burman design and the parameters with significant effects on the production of cholesterol oxidase were identified. Out of the 15 factors screened, agitation speed, cholesterol and yeast extract concentrations had the most significant positive effects on the production of cholesterol oxidase. The optimal levels of these variables and the effects of their mutual interactions on cholesterol oxidase production were determined using Box-Behnken design. Cholesterol oxidase production by Streptomyces anulatus strain NEAE-94 was 11.03, $27.31 \mathrm{U} / \mathrm{mL}$ after Plackett-Burman Design and Box-Behnken design; respectively, with a fold of increase of 6.06 times compared to the production before applying the Plackett-Burman design $(4.51 \mathrm{U} / \mathrm{mL})$.
\end{abstract}

Conclusions: Maximum cholesterol oxidase activity was obtained at the following fermentation conditions: $\mathrm{g} / \mathrm{L}$ (cholesterol 4, yeast extract 5, $\left.\mathrm{NaCl} 0.5, \mathrm{~K}_{2} \mathrm{HPO}_{4} 1, \mathrm{FeSO}_{4} .7 \mathrm{H}_{2} \mathrm{O} 0.01, \mathrm{MgSO}_{4} .7 \mathrm{H}_{2} \mathrm{O} 0.5\right), \mathrm{pH}$, inoculum size $4 \%(\mathrm{v} / \mathrm{v}$ ), temperature $37^{\circ} \mathrm{C}$, agitation speed of $150 \mathrm{rpm}$, medium volume $50 \mathrm{~mL}$ and incubation time 5 days.

Keywords: Streptomyces sp. strain NEAE-94, Identification, 165 rRNA, Plackett-Burman design, Box Behnken design

\footnotetext{
* Correspondence: nouraelahmady@yahoo.com

Department of Bioprocess Development, Genetic Engineering and Biotechnology Research Institute, City for Scientific Research and

Technological Applications, (SRTA-City), Alexandria, Egypt
}

(C) The Author(s). 2020 Open Access This article is licensed under a Creative Commons Attribution 4.0 International License, which permits use, sharing, adaptation, distribution and reproduction in any medium or format, as long as you give appropriate credit to the original author(s) and the source, provide a link to the Creative Commons licence, and indicate if changes were made. The images or other third party material in this article are included in the article's Creative Commons licence, unless indicated otherwise in a credit line to the material. If material is not included in the article's Creative Commons licence and your intended use is not permitted by statutory regulation or exceeds the permitted use, you will need to obtain permission directly from the copyright holder. To view a copy of this licence, visit http://creativecommons.org/licenses/by/4.0/. The Creative Commons Public Domain Dedication waiver (http://creativecommons.org/publicdomain/zero/1.0/) applies to the data made available in this article, unless otherwise stated in a credit line to the data. 


\section{Background}

Cholesterol oxidase (EC 1.1.3.6) is a flavin adenine dinucleotide (FAD)-dependent enzyme that catalyzes cholesterol oxidation to cholestenone (4-cholesten-3one) and hydrogen peroxide [1]. Cholesterol oxidase of microbial origin exhibits a wide range of industrial applications besides to its clinical applications in order to determine food and serum cholesterol levels which are important in the diagnosis of cardiovascular disease, atherosclerosis and other lipid disorders [2, 3]. It plays an essential role in macrophages and leukocytes lysis [4]. In addition, cholesterol oxidase also involved in the manifestation of viral (HIV) disease, bacterial disease (tuberculosis) and Alzheimer's disease [5]. In addition, cholesterol oxidase from Streptomyces natalensis is required for the biosynthesis of the polyene macrolide pimaricin (antifungal antibiotic) which is used as a mould inhibitor in the food industry [6] to prevent food contamination. It is also used as an antibiotic effective in the treatment of keratitis because it interacts with the molecules of sterols present in fungal cell membranes which cause membrane disruption and leading to intracellular components leakage [7].

The Bordetella sp. cholesterol oxidase is used as a promising therapy for lung cancer treatment (both in vitro and in vivo) and led to irreversible cell apoptosis even after addition of cholesterol [8]. Rhodococcus equi infects young horses as well as human immunocompromised patients [9]. Cholesterol oxidase can be used to treat the bacterial infections of Rhodococcus equi for the host cells [10]. Purified bacterial cholesterol oxidase showed strong insecticidal activity against boll weevil larvae "Anthonomus grandis", which decreases the cotton yields [11]. When cholesterol oxidase is ingested by cotton boll weevil larvae, larvae die and the fertility of adult females decreases [12]. Cholesterol oxidase also exhibits insecticidal effect when ingested by pink bollworm (Pectinophora gossypiella), tobacco budworm (Heliothis virescens) and corn earworm (Helicoverpa zea) [13].

The intracellular or extracellular cholesterol oxidase produced by a variety of microorganisms, including Streptomyces natalensis [6] Streptomyces cavourensis [14], Mycobacterium tuberculosis [9], Chromobacterium sp. DS1 [15], Bacillus pumilus W1 and Serratia marcescens W8 [16]. In case of pathogenic bacteria, cholesterol oxidases serve as membrane destroying agents and thus contribute to their pathogenicity as a virulence factor [9]. Non-pathogenic bacteria use cholesterol for growth as a carbon and energy sources.

Many scientists have sought to increase the yield of cholesterol oxidase because of low production by several microorganisms. The production of cholesterol oxidase is greatly influenced by the soluble component of the medium and culture conditions, including temperature, incubation time, $\mathrm{pH}$, inoculum size and the rate of agitation [17]. Response surface methodology (RSM) is an effective tool by which the optimum conditions can be determined for a multivariate system. Statistical methods were more effective than the classical method (one-variable-at-a-time) in determining the optimal concentrations of medium components used to produce cholesterol oxidase. Statistical methods offer a range of advantages, including lower experimental numbers, suitability for multi-factor experiments, studying the mutual interactions among the variables, and identifying the most appropriate conditions for maximum enzyme production [18]. Statistical optimization by surface response methodology involves two steps, firstly, the screening of significant factors and, secondly, the optimization of such factors [19].

The objectives of this study were to identify robust cholesterol-assimilating actinomycetes strain with high cholesterol oxidase activity, and to optimize the fermentation conditions for enhanced production of cholesterol oxidase by Streptomyces anulatus strain NEAE-94 using response surface methodology.

\section{Results}

Actinomycete strain, Streptomyces sp. strain NEAE-94, has been tested for its cholesterol oxidase activity with a plate-based method, the formation of the pink areas around the colonies indicated the presence of cholesterol oxidase activity (Fig. 1). The process of cholesteroloxidation is the oxidation of cholesterol with the use of cholesterol oxidase to 4-cholesten-3-one and hydrogen peroxide. The hydrogen peroxide generated by cholesterol oxidation was then combined with 4-aminoantipyrine and phenol by peroxidase to produce quinoneimine coloration (Supplementary Figure 1). The promising strain was identified based on morphological, cultural, physiological and chemotaxonomic characteristics, in addition to $16 \mathrm{~S}$ rRNA sequence.

Cultural characteristics of Streptomyces sp. strain NEAE-94 The isolate showed well growth on four different media, including oatmeal agar, inorganic salt-starch agar, tyrosine agar and yeast malt extract agar. Weak growth was observed on glycerol asparagines agar and peptone-yeast extract iron agar (Supplementary Table 1). Aerial mycelium color was whitish yellow on yeast extract-malt extract agar (Fig. 2a); yellow on oatmeal agar, tyrosine agar and inorganic salt-starch agar (Fig. 2b), while is faint yellow on peptone-yeast extract iron agar and glycerol asparagines agar. However, the substrate mycelium develop a yellow color on oatmeal agar, inorganic saltstarch agar, yeast extract -malt extract agar; brownish orange color on tyrosine agar media. Whereas, a faint orange color was developed on both glycerol asparagine 


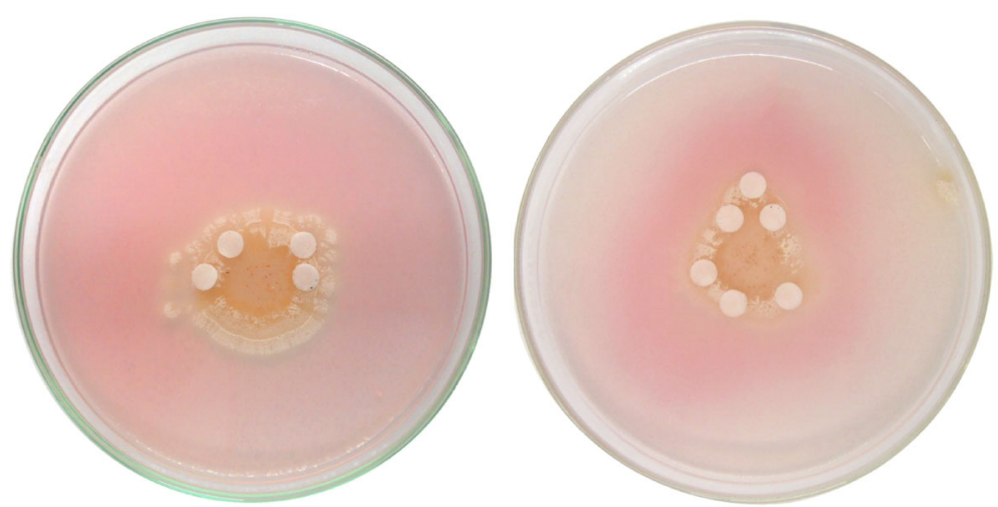

Fig. 1 Cholesterol oxidase production of Streptomyces sp. NEAE-94 detected by plate assay method

agar and peptone-yeast extract iron agar media. A yellow diffusible pigment was produced in inorganic salt-starch agar, yeast extract-malt extract agar, tyrosine agar and glycerol asparagine agar; a faint yellow pigment was produced in oatmeal agar. The diffusible pigment was not $\mathrm{pH}$ indicator. No pigments were produced in peptoneyeast extract iron agar.

\section{Physiological properties of Streptomyces sp. strain NEAE-} 94

The physiological properties of Streptomyces sp. strain NEAE-94 are listed in Table 1. It utilized trehalose, $\mathrm{D}(+)$ xylose, $\mathrm{D}(+)$ mannose, rhamnose, $\mathrm{D}(+)$ galactose, raffinose, $\mathrm{D}(-)$ fructose, $\mathrm{D}(+)$ glucose, L-arabinose, sucrose, maltose and cellulose, but could not utilized ribose as

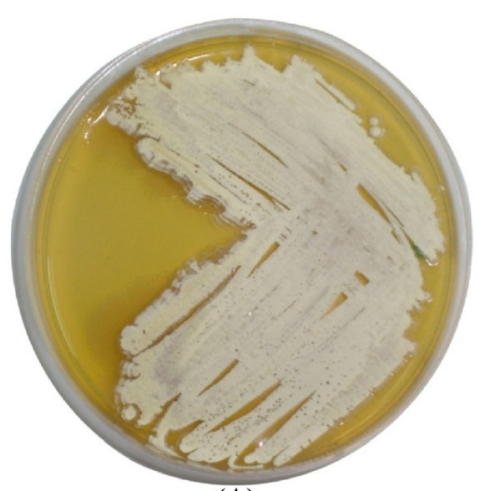

(A)

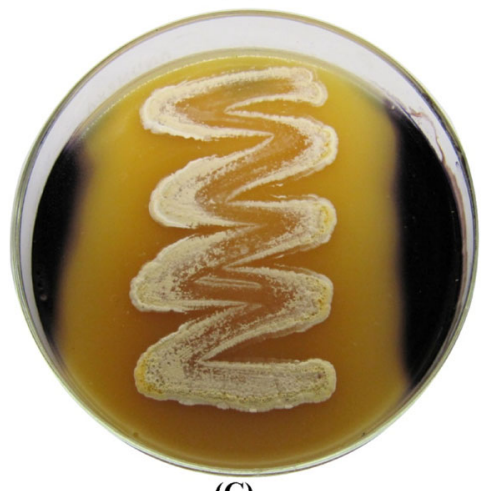

(C)

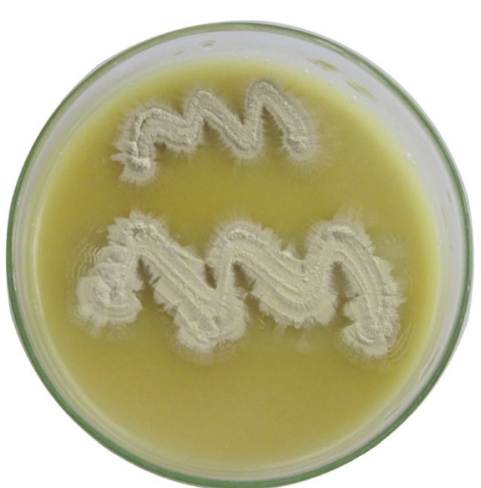

(B)

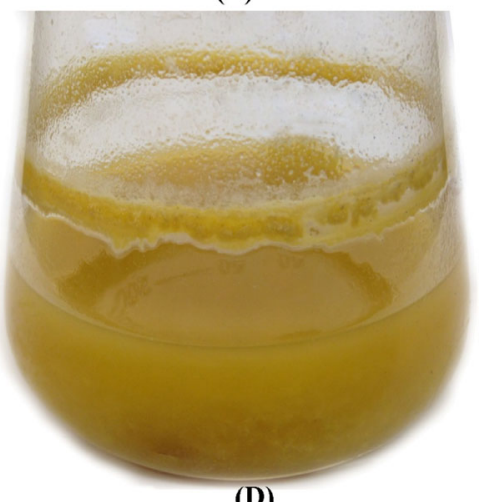

(D)

Fig. 2 The colored photograph of Streptomyces sp. NEAE-94 aerial mycelium after growth on yeast extract -malt extract agar (a) and inorganic salt-starch agar media (b) at $30^{\circ} \mathrm{C}$ for 7 days, (c) plate assay showing starch hydrolysis by Streptomyces sp. strain NEAE-94 and (d) cholesterol oxidase production in submerged fermentation 
Table 1 Phenotypic properties of Streptomyces sp. strain NEAE-94 and related Streptomyces species. Reference species properties have been taken from Bergey's Manual of Systematic Bacteriology [20]

\begin{tabular}{|c|c|c|c|c|c|}
\hline Characteristic & $\begin{array}{l}\text { Streptomyces } \\
\text { sp. strain NEAE-94 }\end{array}$ & $\begin{array}{l}\text { Streptomyces } \\
\text { anulatus }\end{array}$ & $\begin{array}{l}\text { Streptomyces } \\
\text { flavofuscus }\end{array}$ & $\begin{array}{l}\text { Streptomyces } \\
\text { fimicarius }\end{array}$ & $\begin{array}{l}\text { Streptomyces } \\
\text { parvus }\end{array}$ \\
\hline $\begin{array}{l}\text { Aerial } \\
\text { mycelium on ISP medium } 2\end{array}$ & Whitish -yellow & Yellow or white & $\begin{array}{l}\text { Yellow, } \\
\text { green- } \\
\text { yellow }\end{array}$ & $\begin{array}{l}\text { Yellow } \\
\text { or white }\end{array}$ & Yellow \\
\hline Diffusible pigment & Yellow & Trace of yellow & $\begin{array}{l}\text { Yellow- } \\
\text { gray-brown }\end{array}$ & & Yellow \\
\hline Substrate mycelium on ISP medium 2 & Yellow & $\begin{array}{l}\text { Pale yellow; grayish } \\
\text { yellow or yellowish } \\
\text { brown }\end{array}$ & $\begin{array}{l}\text { Yellow- } \\
\text { gray-brown }\end{array}$ & $\begin{array}{l}\text { Not } \\
\text { distinctive }\end{array}$ & $\begin{array}{l}\text { Not } \\
\text { distinctive }\end{array}$ \\
\hline Surface of spores & Smooth & Smooth & Smooth & Smooth & Smooth \\
\hline Morphology of spore chain & Rectiflexibles & Rectiflexibiles & Rectiflexibiles & Rectiflexibiles & Rectiflexibiles \\
\hline $\begin{array}{l}\text { Melanin production on Tyrosine agar, } \\
\text { peptone-yeast extract iron agar or } \\
\text { tryptone-yeast extract broth }\end{array}$ & - & - & - & - & - \\
\hline \multicolumn{6}{|l|}{ Utilization of carbon sources } \\
\hline $\begin{array}{l}\mathrm{D}(+) \text { glucose, } \mathrm{D}(-) \text { fructose, } L \text {-arabinose, } \\
\mathrm{D}(+) \text { xylose, rhamnose }\end{array}$ & + & + & & + & + \\
\hline Sucrose, raffinose & + & + & & \pm & \pm \\
\hline $\mathrm{D}(+)$ mannose, cellulose & + & + & & + & \\
\hline Trehalose, $\mathrm{D}(+)$ galactose, maltose & + & & & & \\
\hline Ribose & - & & & & \\
\hline
\end{tabular}

Abbreviations: + Positive, - Negative, \pm Doubtful, Blank cells no data available. L-asparaginase, gelatinase, cellulose, protease and a-amylase were produced by Streptomyces sp. strain NEAE-94 while chitosanase, lecithinase and uricase were not produced. Maximum $\mathrm{NaCl}$ tolerance $(5 \%$, w/v).The optimal growth temperature was $30^{\circ} \mathrm{C}$ and optimal pH was 7.0. It exhibited positive antimicrobial activities against Staphylococcus aureus, E. coli, Bacillus subtilis and Pseudomonas aeruginosa. Nitrate reduction, coagulation and peptonization of milk were positive

the sole carbon source. Streptomyces sp. strain NEAE-94 has reduced nitrate to nitrite. Coagulation and peptonization of milk were positive. Production of protease, $\alpha-$ amylase (Fig. 2c), cellulase, gelatinase and asparaginase was positive. On the other hand, production of uricase, lecithinase and chitosanase was negative. The optimal growth temperature was $30^{\circ} \mathrm{C}$ and the optimal $\mathrm{pH}$ was 7.0. Streptomyces sp. strain NEAE-94 grew in the presence of $\mathrm{NaCl}$ up to $5 \%(\mathrm{w} / \mathrm{v})$. Streptomyces sp. strain NEAE-94 showed positive antimicrobial activities against Staphylococcus aureus, E. coli, Bacillus subtilis and Pseudomonas aeruginosa, but no activities were shown against Candida albicans, Rhizoctonia solani, Aspergillus niger, Fusarium oxysporum, Alternaria solani, Sacchromyces cerevisiae, Bipolaris oryzae or Klebsiella pneumonia. Streptomyces sp. strain NEAE-94 did not produce melanoid pigments in tyrosine agar, peptone-yeast extract iron agar or tryptone-yeast extract broth.

\section{Morphological features of Streptomyces sp. strain NEAE- 94}

Morphological characteristics of Streptomyces sp. strain NEAE-94 was observed by scanning electron micrograph after incubation on medium of starch nitrate agar medium at $30^{\circ} \mathrm{C}$ for 14 days. Microscopic observation of Streptomyces sp. strain NEAE-94 showed rectiflexibiles spores chains (Fig. 3). In general, chains of mature spores are long. Spore shape is elongated $(0.593-0.754 \times$ $0.995-1.341 \mu \mathrm{m})$, irregular and the spore surface is smooth (Fig. 3).

\section{S rRNA gene sequence analysis and phylogenetic analysis}

The obtained 16S rRNA sequence of Streptomyces sp. strain NEAE-94 was determined which gave an almost complete sequence with $1536 \mathrm{bp}$ and further subjected to the BLAST search of the GenBank database and the results showed homologies with other relevant sequences of many species belonging to the Streptomyces genus. The phylogenetic tree (Fig. 4) showed that Streptomyces sp. strain NEAE-94 shared gene similarity of 99.38\% to that of Streptomyces anulatus strain BZ10-24, query cover 94\% (GenBank accession no. KC493992.1); $99.59 \%$ to that of Streptomyces parvus strain 3151, query cover 94\% (GenBank accession no. EF063462.1); 99.38\% to that of Streptomyces flavofuscus strain NRRL B-2594, query cover 94\% (GenBank accession no. EF178690.1) and $99.19 \%$ to that of Streptomyces fimicarius strain BWL-H1, query cover 95\% (GenBank accession no. MG197994.1).

The whole morphological and physiological properties of Streptomyces sp. strain NEAE-94 and its closest 


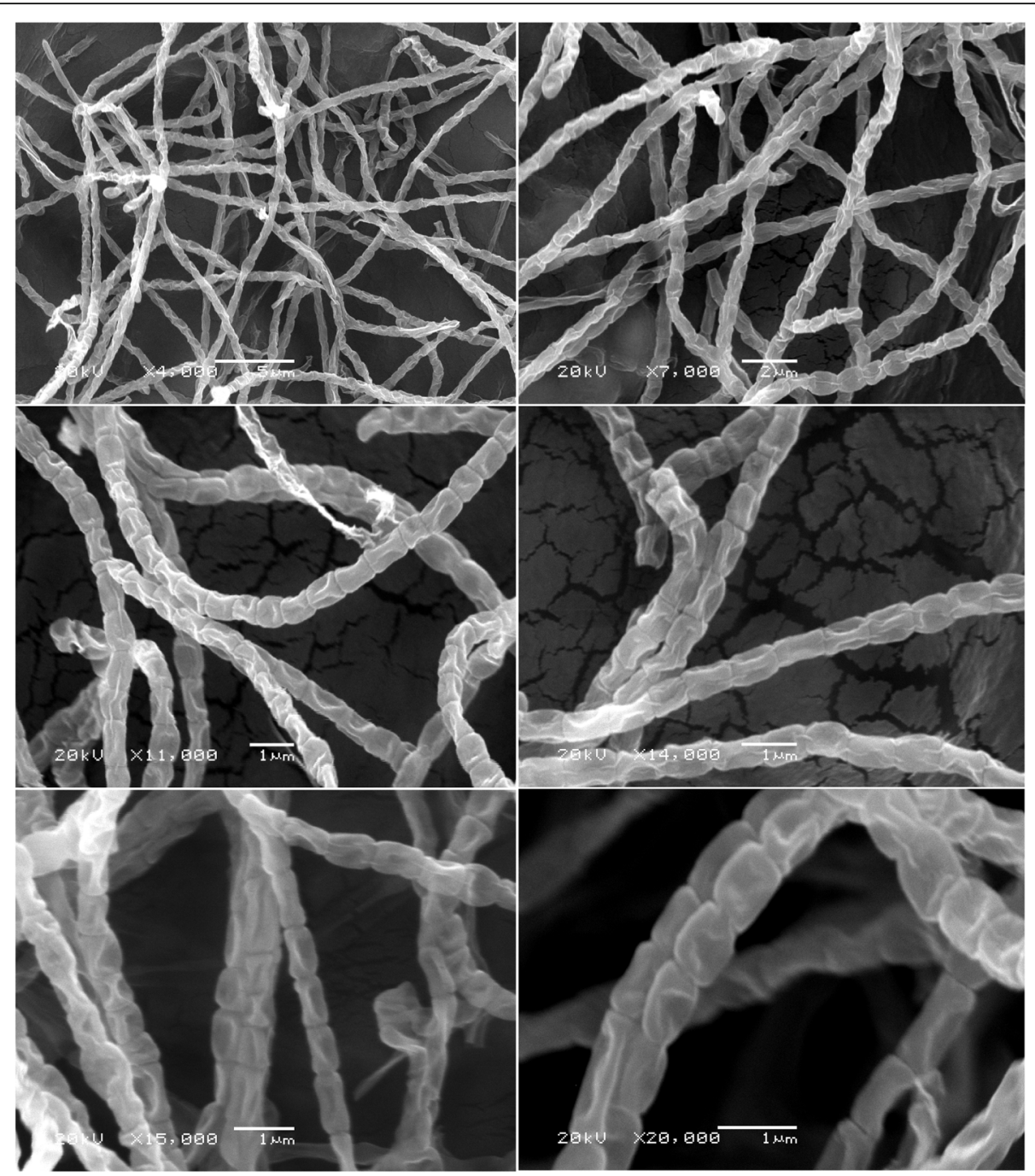

Fig. 3 Spore-chain and spore-surface morphology of Streptomyces sp. strain NEAE-94 under scanning electron microscope at magnification of $4000 X-20,000 X$

phylogenetic neighbors of the genus Streptomyces which showed significant similarities are shown in Table 1. The strain (Streptomyces anulatus) has been deposited in the Culture Collection Ain Shams University (CCASU). The culture collection accession number CCASU 20202 is assigned to the deposited strain.

\section{Screening of significant factors for production of cholesterol oxidase using Plackett-Burman design}

The design matrix used for screening of the significant factors for production of cholesterol oxidase and the appropriate responses are shown in Table 2. The mycelial growth of Streptomyces anulatus strain NEAE-94 during cholesterol oxidase production in shake flask in submerged fermentation is shown in Fig. 2d. The results showed broad variability of the cholesterol oxidase activity $(0.87$ to $11.03 \mathrm{U} / \mathrm{mL})$ reflecting the significance of the medium optimization for enhanced cholesterol oxidase production. The highest production of cholesterol oxidase $(11.03 \mathrm{U} / \mathrm{mL})$ was achieved in the run no. 18 using $50 \mathrm{~mL}$ medium $/ 250 \mathrm{~mL}$ conical flask consists of $(\mathrm{g} / \mathrm{L})$ : Glucose 5; starch 10; cholesterol 3; yeast extract 4; peptone $4 ;\left(\mathrm{NH}_{4}\right)_{2} \mathrm{SO}_{4} 4 ; \mathrm{FeSO}_{4} .7 \mathrm{H}_{2} \mathrm{O} 0.01$; $\mathrm{MgSO}_{4} .7 \mathrm{H}_{2} \mathrm{O} 0.5 ; \mathrm{NaCl} 0.5 ; \mathrm{K}_{2} \mathrm{HPO}_{4} 1$ and $\mathrm{pH}$ 7; inoculum size was $4 \%(\mathrm{v} / \mathrm{v})$ and incubated for 5 days at $37^{\circ} \mathrm{C}$ using an agitation speed of $150 \mathrm{rpm}$. In the run no. 4, the lowest production of cholesterol oxidase was obtained $(0.87 \mathrm{U} / \mathrm{mL})$ using $50 \mathrm{~mL}$ medium $/ 250 \mathrm{~mL}$ conical flask consists of (g/ L): Glucose 5; starch 10; cholesterol 1; yeast extract 1; peptone 1; $\left(\mathrm{NH}_{4}\right)_{2} \mathrm{SO}_{4} 4 ; \mathrm{FeSO}_{4} .7 \mathrm{H}_{2} \mathrm{O} 0.05 ; \mathrm{MgSO}_{4} .7 \mathrm{H}_{2} \mathrm{O} 0.1$; $\mathrm{NaCl} 1 ; \mathrm{K}_{2} \mathrm{HPO}_{4} 1$ and $\mathrm{pH}$ 9; inoculum size was $4 \%(\mathrm{v} / \mathrm{v})$ and incubated for 7 days at $37^{\circ} \mathrm{C}$ using an agitation speed of $100 \mathrm{rpm}$.

Table 3 shows the statistical analysis of the results of the Plackett-Burman design. Figure 5a shows the main 


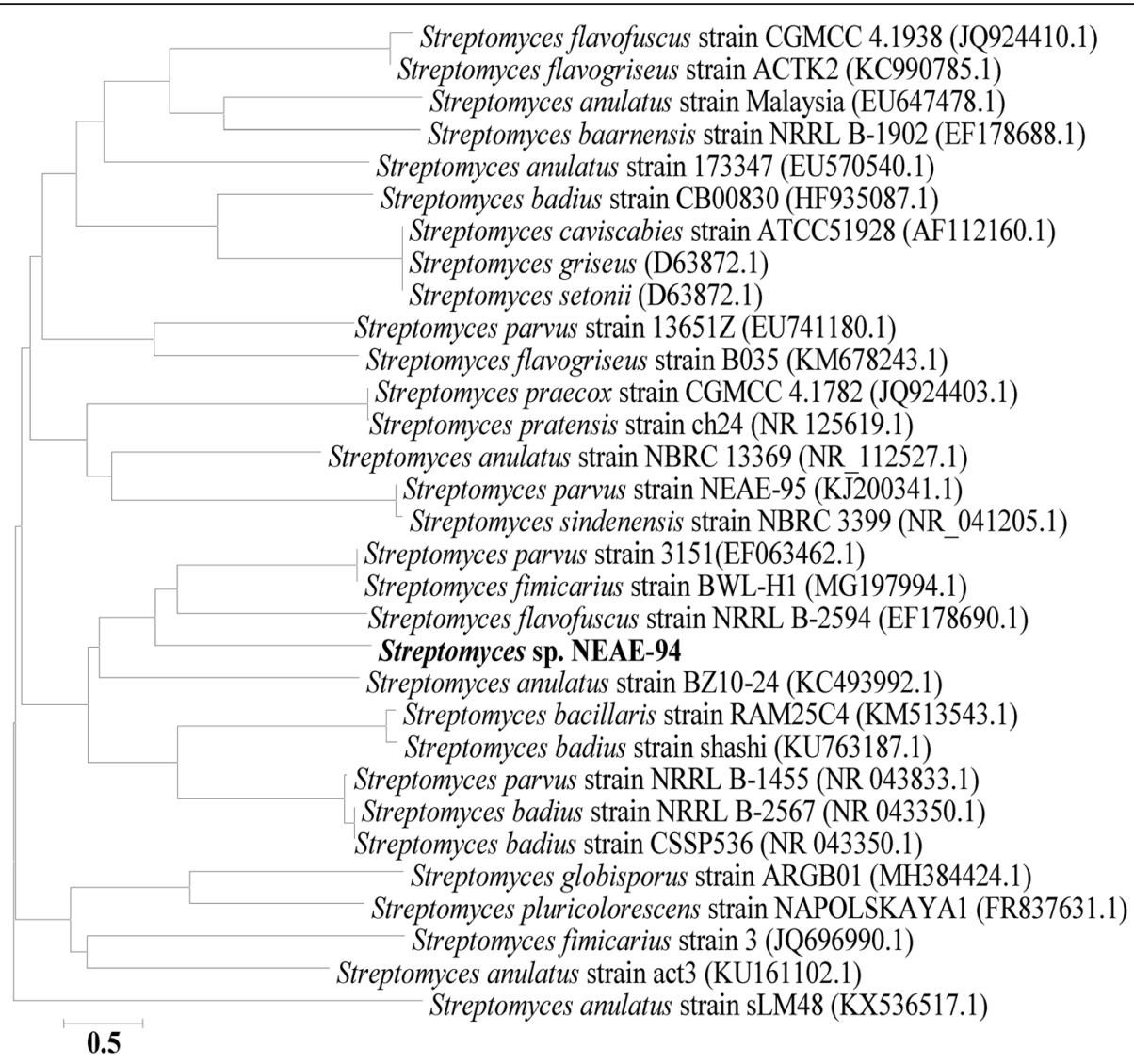

Fig. 4 Phylogenetic tree obtained by Neighbour-joining method showing the relationship between Streptomyces sp. strain NEAE-94 and other related species of Streptomyces based on the 16S rRNA sequences

effect of the individual independent factors on the cholesterol oxidase production. Figure $5 \mathrm{a}$ revealed that, temperature, agitation speed, $\mathrm{pH}$, starch, cholesterol, peptone, yeast extract, ammonium sulphate and $\mathrm{K}_{2} \mathrm{HPO}_{4}$ positively affect cholesterol oxidase production, whereas the remaining factors named incubation time, glucose, inoculum size, $\mathrm{MgSO}_{4}, \mathrm{NaCl}$ and $\mathrm{FeSO}_{4}$ negatively affect cholesterol oxidase production. The data revealed that, starch $(\mathrm{G})$, peptone $(\mathrm{K})$ and ammonium sulphate (L) with higher $P$-values $(0.9271,0.9573$, 0.9370; respectively), lower effects $(0.09,0.05$ and 0.07 ; respectively) and lower contribution \% (0.04, 0.02 and 0.04; respectively) are insignificant factors. The Pareto chart shows absolute effects values and illustrates the significance order of the factors that influence cholesterol oxidase production. The Pareto chart shows a reference line, any absolute effect value extending past this reference line is highly essential (Fig. $5 b$ ). Figure $5 \mathrm{c}$ displays a normal probability plot of the residuals. Figure $5 \mathrm{~d}$ shows the plot of the predicted cholesterol oxidase production versus actual values.

The values of the determination coefficient $\left(R^{2}=\right.$ 0.9996) and the adjusted determination coefficient (Adj.
$\left.\mathrm{R}^{2}=0.9978\right)$ are very high. Cholesterol, agitation speed with a $P$-value of $<0.0001$ was determined to be the most significant factors, followed by the concentration of yeast extract, incubation time (0.0001) then glucose (0.0002) (Table 3). The $P$-value $<0.05$ (0.0001) implies that the model terms are significant. The $F$-value of the model (573.21) means that it is significant. The first order polynomial equation representing the production of cholesterol oxidase in relation to the independent factors was obtained by neglecting the insignificant factors:

$$
\begin{aligned}
\mathrm{Y}= & 5.17+0.15 \mathrm{~A}-0.88 \mathrm{~B}-0.45 \mathrm{C}+1.01 \mathrm{D} \\
& +0.42 \mathrm{E}-0.78 \mathrm{~F}+2.18 \mathrm{H}+0.95 \mathrm{~K} \\
& +0.17 \mathrm{M}-0.41 \mathrm{~N}-0.11 \mathrm{O}-0.19 \mathrm{P}
\end{aligned}
$$

Where $\mathrm{Y}$ is the production of cholesterol oxidase and $\mathrm{A}, \mathrm{B}, \mathrm{C}, \mathrm{D}, \mathrm{E}, \mathrm{F}, \mathrm{H}, \mathrm{K}, \mathrm{M}, \mathrm{N}, \mathrm{O}, \mathrm{P}$ are temperature, the time of incubation, size of inoculum, speed of agitation, $\mathrm{pH}$, concentration of glucose, concentration of cholesterol, concentration of yeast extract, concentration of $\mathrm{K}_{2} \mathrm{HPO}_{4}, \mathrm{NaCl}, \mathrm{MgSO}_{4}$ and $\mathrm{FeSO}_{4}$; respectively.

For assessment of Plackett-Burman design precision, the following production medium was used for 


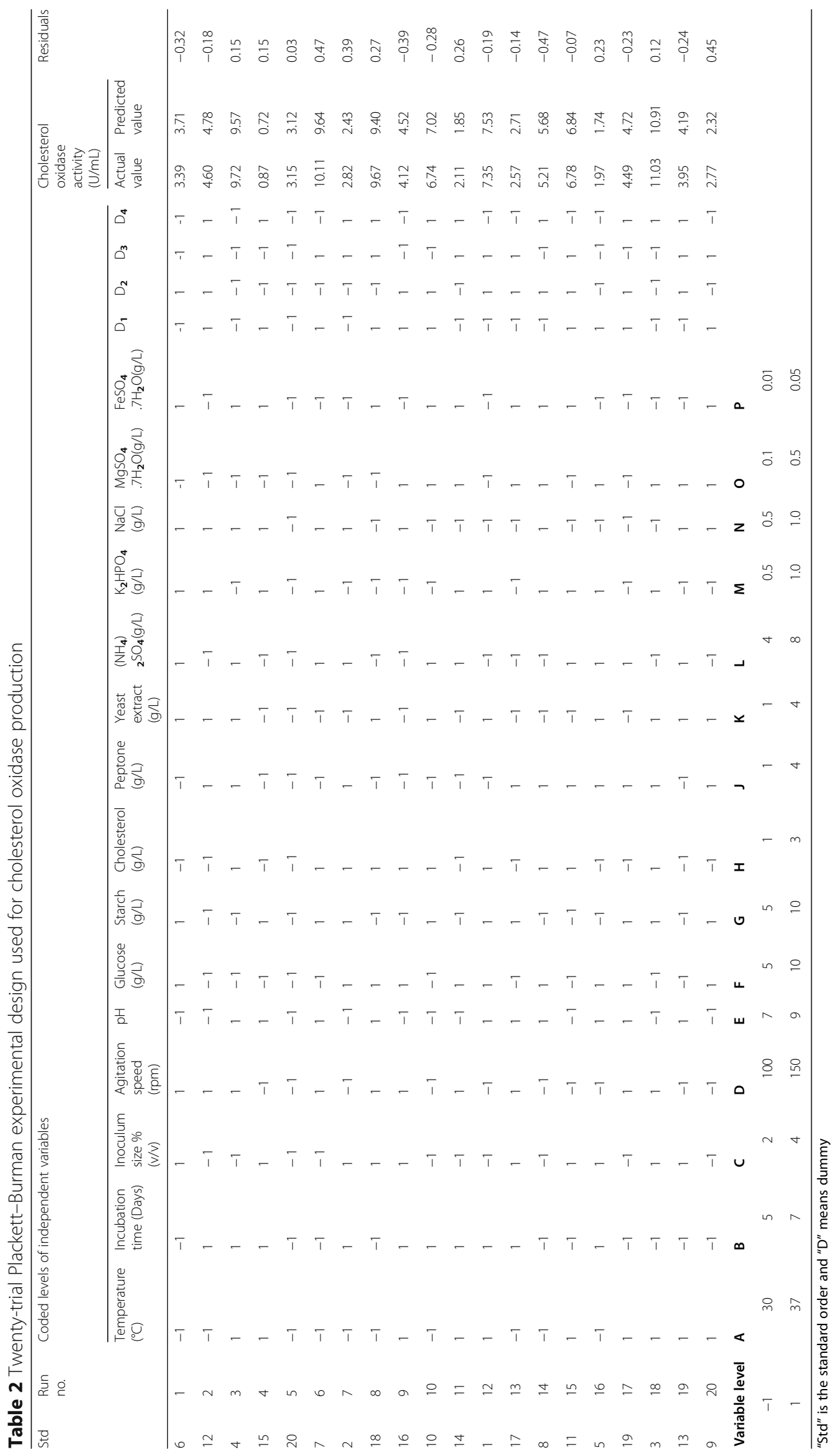


Table 3 Statistical analysis of Plackett-Burman design

\begin{tabular}{|c|c|c|c|c|c|}
\hline Term & Coefficient & Effect & $\%$ Contribution & $F$-value & $\begin{array}{l}\boldsymbol{P} \text {-value } \\
\text { Prob }>\boldsymbol{F}\end{array}$ \\
\hline Intercept & 5.17 & & & 573.21 & $0.0001^{*}$ \\
\hline A-Temperature & 0.15 & 0.30 & 0.25 & 17.15 & $0.0256^{*}$ \\
\hline B-Incubation time & -0.88 & -1.77 & 8.86 & 615.20 & $0.0001^{*}$ \\
\hline C-Inoculum size & -0.45 & -0.91 & 2.33 & 161.94 & $0.0010^{*}$ \\
\hline D-Agitation speed & 1.01 & 2.02 & 11.58 & 804.04 & $<0.0001^{*}$ \\
\hline E-pH & 0.42 & 0.84 & 2.00 & 138.84 & $0.0013^{*}$ \\
\hline F-Glucose & -0.78 & -1.56 & 6.92 & 480.30 & $0.0002^{*}$ \\
\hline G-Starch & 0.04 & 0.09 & 0.02 & $9.87 \times 10^{-3}$ & $0.9271^{*}$ \\
\hline $\mathrm{H}$-Cholesterol & 2.18 & 4.37 & 54.13 & 3757.27 & $<0.0001^{*}$ \\
\hline J-Peptone & 0.02 & 0.05 & 0.01 & $3.38 \times 10^{-3}$ & 0.9573 \\
\hline K-Yeast extract & 0.95 & 1.90 & 10.20 & 707.66 & $0.0001^{*}$ \\
\hline $\mathrm{L}-\left(\mathrm{NH}_{4}\right)_{2} \mathrm{SO}_{4}$ & 0.04 & 0.07 & 0.02 & $7.37 \times 10^{-3}$ & 0.9370 \\
\hline $\mathrm{M}-\mathrm{K}_{2} \mathrm{HPO}_{4}$ & 0.17 & 0.34 & 0.33 & 22.89 & $0.0174^{*}$ \\
\hline $\mathrm{N}-\mathrm{NaCl}$ & -0.41 & -0.83 & 1.95 & 135.29 & $0.0014^{*}$ \\
\hline O- $\mathrm{MgSO}_{4} \cdot 7 \mathrm{H}_{2} \mathrm{O}$ & -0.11 & -0.23 & 0.14 & 9.98 & 0.0509 \\
\hline P- $\mathrm{FeSO}_{4} \cdot 7 \mathrm{H}_{2} \mathrm{O}$ & -0.19 & -0.38 & 0.40 & 27.95 & $0.0132^{*}$ \\
\hline PRESS & 12.92 & & Adeq Precision & & 79.34 \\
\hline C.V. $\%$ & 3.08 & & Pred $R^{2}$ & & 0.9261 \\
\hline Mean & 5.17 & & Adj $R^{2}$ & & 0.9978 \\
\hline Std. Dev. & 0.16 & & $R^{2}$ & & 0.9996 \\
\hline
\end{tabular}

* Significant values, "F: Fishers's function, PRESS is the predicted residual sum of squares, C.V. \% is the coefficient of variation\%"

cholesterol oxidase production (g/L): $\mathrm{FeSO}_{4} .7 \mathrm{H}_{2} \mathrm{O}$ 0.01; $\mathrm{MgSO}_{4} .7 \mathrm{H}_{2} \mathrm{O} 0.5 ; \mathrm{NaCl} 0.5 ; \mathrm{K}_{2} \mathrm{HPO}_{4} 1$; yeast extract 4; cholesterol 3 and $\mathrm{pH}$ 7. The production medium was inoculated with inoculum size of $4 \%(\mathrm{v} / \mathrm{v})$ and incubated at a temperature of $37^{\circ} \mathrm{C}$ in a shaker incubator at 150 rpm for 5 days. The production of cholesterol oxidase using the previous medium was $11.03 \mathrm{U} / \mathrm{mL}$, which was increased 2.45 times compared to the enzyme activity obtained before application of Plackett-Burman design (4.51 U/mL).

\section{Optimization of the selected significant variables by Box- Behnken design}

Box-Behnken design was used to obtain the optimal levels of the most significant factors influencing cholesterol oxidase production by Streptomyces anulatus strain NEAE94 and to study the interaction effects among these factors. In the current study, 15 experiments with various combinations of agitation speed, cholesterol concentration and yeast extract concentration were performed and the experimental and predicted cholesterol oxidase production and residuals for the 15 trials are provided in Table 4.

Based on the variations in the agitation speed, cholesterol concentration and yeast extract concentration, the results showed variations in the cholesterol oxidase production. Cholesterol oxidase production ranged from 5.64-27.31 U/mL. The lowest cholesterol oxidase production by Streptomyces anulatus strain NEAE-94 (5.64 $\mathrm{U} / \mathrm{mL}$ ) was achieved in the 13th run when the agitation speed was $100 \mathrm{rpm}$, cholesterol concentration was $4 \mathrm{~g} / \mathrm{L}$ and yeast extract concentration was $3 \mathrm{~g} / \mathrm{L}$. The maximum value of cholesterol oxidase production was achieved in the 12th run with value of $27.31 \mathrm{U} / \mathrm{mL}$, when agitation speed was $150 \mathrm{rpm}$, cholesterol concentration was $4 \mathrm{~g} / \mathrm{L}$ and yeast extract concentration was $5 \mathrm{~g} / \mathrm{L}$.

\section{The analysis of variance (ANOVA) for multiple regression analysis}

Table 5 contains multiple regression analysis and ANOVA for the results of the Box-Behnken design. The ANOVA of the multiple regression analysis show the model to be highly significant, as can be seen from the low probability value $(<0.0001)$ and the value of Fisher's F-test $(113.82)$ (Table 5). The current $R^{2}$ and adjusted $R^{2}$ values are 0.9951 and 0.9864 ; respectively. While, predicted $R^{2}$ value is 0.9427 . Accuracy and reliability of the model can be seen in the small percentage of the coefficient of variation value $(\mathrm{CV}=5.44 \%)$, mean value (15.16), adequate precision value (31.469), PRESS value (40.03) and standard deviation value (0.82) (Table 5). 

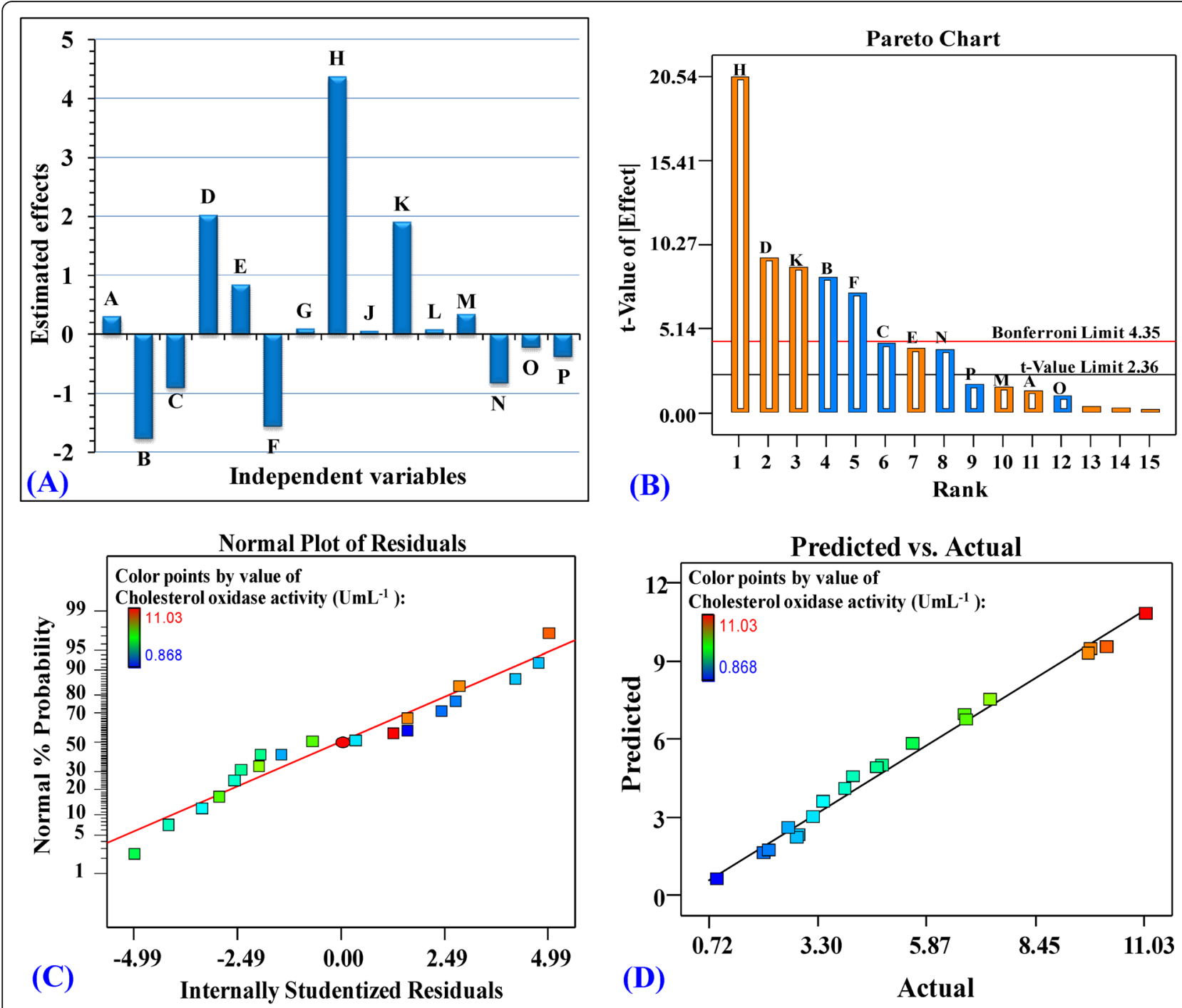

Fig. $\mathbf{5}$ a The main effects of the variables, $\mathbf{b}$ The Pareto chart shows the order of significance of each variable, $\mathbf{c}$ The normal probability plot of the residuals, $\mathbf{d}$ Correlation between the experimented and predicted values for cholesterol oxidase production by Streptomyces anulatus strain NEAE-94 determined by the first-order polynomial equation

The significance of each coefficient was defined in terms of both $P$ and $F$ values listed in Table 5. It can be seen from the $P$-values and $F$-values that the linear coefficients of cholesterol concentration, interaction between the agitation speed and cholesterol concentration; agitation speed and yeast extract concentration; cholesterol concentration and yeast extract concentration and quadratic effects of agitation speed, cholesterol concentration and yeast extract concentration are significant as it is evident from the $F$-values of $119.05,7.80,62.24,7.80$, $321.59,79.45,526.73$; respectively, and $P$-values of $0.0001,0.0383,0.0005,0.0383,<0.0001,0.0003$, < 0.0001 ; respectively. On the other hand, $P$-values of the linear coefficients of agitation speed $\left(\mathrm{X}_{1}\right)$ and yeast extract concentration $\left(\mathrm{X}_{3}\right)$ indicate that they had nonsignificant effects on cholesterol oxidase production by the strain under study.

The quadratic model of Box-Behnken design used for cholesterol oxidase production by Streptomyces anulatus strain NEAE-94, with a non-significant lack of fit $(F$-value 1.51 and $P$-value $=0.4219)$ and a very low $P$-value $<0.0001$ was shown in the fit summary results (Supplementary Table 2). The largest adjusted and predicted $R^{2}$ of 0.9864 and 0.9427 and the lowest standard deviation (0.82) was reported in the summary statistics of the quadratic model.

The optimum levels of agitation speed, cholesterol concentration and yeast extract concentration giving the maximum cholesterol oxidase production was evaluated by a second-order polynomial equation. Cholesterol 
Table 4 Three-level Box-Behnken design representing cholesterol oxidase production as influenced by agitation speed $\left(X_{1}\right)$, cholesterol concentration $\left(X_{2}\right)$ and yeast extract concentration $\left(X_{3}\right)$

\begin{tabular}{|c|c|c|c|c|c|c|c|c|c|c|}
\hline \multirow[t]{2}{*}{ Std } & \multirow[t]{2}{*}{ Run } & \multicolumn{2}{|c|}{ Agitation speed } & \multicolumn{2}{|c|}{ Cholesterol concentration } & \multicolumn{2}{|c|}{ Yeast extract concentration } & \multicolumn{2}{|c|}{ Cholesterol oxidase activity $(\mathrm{U} / \mathrm{mL})$} & \multirow[t]{2}{*}{ Residuals } \\
\hline & & Coded & Actual (rpm) & Coded & Actual $(g / L)$ & Coded & Actual $(g / L)$ & Experimental & Predicted & \\
\hline 15 & 1 & 0 & 150 & 0 & 4 & 0 & 5 & 26.44 & 26.55 & -0.10 \\
\hline 13 & 2 & 0 & 150 & 0 & 4 & 0 & 5 & 25.88 & 26.55 & -0.66 \\
\hline 1 & 3 & -1 & 100 & -1 & 2 & 0 & 5 & 12.90 & 12.97 & -0.07 \\
\hline 9 & 4 & 0 & 150 & -1 & 2 & -1 & 3 & 10.31 & 10.50 & -0.19 \\
\hline 2 & 5 & 1 & 200 & -1 & 2 & 0 & 5 & 11.48 & 10.74 & 0.74 \\
\hline 7 & 6 & -1 & 100 & 0 & 4 & 1 & 7 & 13.13 & 12.58 & 0.55 \\
\hline 3 & 7 & -1 & 100 & 1 & 6 & 0 & 5 & 16.29 & 17.03 & -0.74 \\
\hline 12 & 8 & 0 & 150 & 1 & 6 & 1 & 7 & 17.76 & 17.57 & 0.19 \\
\hline 11 & 9 & 0 & 150 & -1 & 2 & 1 & 7 & 8.44 & 8.91 & -0.47 \\
\hline 10 & 10 & 0 & 150 & 1 & 6 & -1 & 3 & 15.03 & 14.56 & 0.47 \\
\hline 4 & 11 & 1 & 200 & 1 & 6 & 0 & 5 & 19.47 & 19.40 & 0.07 \\
\hline 14 & 12 & 0 & 150 & 0 & 4 & 0 & 5 & 27.31 & 26.55 & 0.77 \\
\hline 5 & 13 & -1 & 100 & 0 & 4 & -1 & 3 & 5.64 & 5.38 & 0.27 \\
\hline 6 & 14 & 1 & 200 & 0 & 4 & -1 & 3 & 11.40 & 11.95 & -0.55 \\
\hline 8 & 15 & 1 & 200 & 0 & 4 & 1 & 7 & 5.89 & 6.16 & -0.27 \\
\hline
\end{tabular}

"Std" is the standard order

oxidase production can be predicted by applying the following second-order regression equation in terms of the independent variables:

$$
\begin{aligned}
\mathrm{Y}= & 26.55+0.04 \mathrm{X}_{1}+3.18 \mathrm{X}_{2}+0.35 \mathrm{X}_{3} \\
& +1.15 \mathrm{X}_{1} \mathrm{X}_{2}-3.25 \mathrm{X}_{1} \mathrm{X}_{3} \\
& +1.15 \mathrm{X}_{2} \mathrm{X}_{3}-7.69 \mathrm{X}_{1}^{2}-3.82 \mathrm{X}_{2}^{2}-9.84 \mathrm{X}_{3}^{2}
\end{aligned}
$$

Where $\mathrm{Y}$ is the cholesterol oxidase production, $\mathrm{X}_{1}$ is the coded value of agitation speed, $\mathrm{X}_{2}$ is the coded value of cholesterol concentration and $\mathrm{X}_{3}$ is the coded value of yeast extract concentration.

\section{Three dimensional (3D) surface and contour plots}

To understand the interaction among the three factors $\left(\mathrm{X}_{1}-\mathrm{X}_{3}\right)$ and the optimum level of each factor required for the maximum cholesterol oxidase production, the 3D curves and its corresponding contour plots were generated by plotting the cholesterol oxidase production on the $\mathrm{Z}$ axis versus two factors are allowed to vary and the

Table 5 Regression statistics and analysis of variance (ANOVA) for Box-Behnken design results

\begin{tabular}{lllll}
\hline Source & $\boldsymbol{d f}$ & Coefficient estimate & $\boldsymbol{F}$-value & $\boldsymbol{P}$-value Prob $>\boldsymbol{F}$ \\
\hline Model & 9 & 26.55 & 113.82 & $<0.0001^{*}$ \\
$X_{1}$ & 1 & 0.04 & 0.02 & 0.9071 \\
$X_{2}$ & 1 & 3.18 & 119.05 & $0.0001^{*}$ \\
$X_{3}$ & 1 & 0.35 & 1.48 & 0.2782 \\
$X_{1} X_{2}$ & 1 & 1.15 & 7.80 & $0.0383^{*}$ \\
$X_{1} X_{3}$ & 1 & -3.25 & 62.24 & $0.0005^{*}$ \\
$X_{2} X_{3}$ & 1 & 1.15 & 7.80 & $0.0383^{*}$ \\
$X_{1}^{2}$ & 1 & -7.69 & 321.59 & $<0.0001^{*}$ \\
$X_{2}^{2}$ & 1 & -3.82 & 79.45 & $0.0003^{*}$ \\
$X_{3}^{2}$ & 1 & -9.84 & 526.73 & $<0.0001^{*}$ \\
$R^{2}$ & 0.9951 & Std. Dev. & 0.82 & 15.16 \\
Adj R & 0.9864 & Mean & 5.44 & \\
Pred R & 0.9427 & C.V. $\%$ & 40.03 & \\
Adeq Precision & 31.469 & PRESS & &
\end{tabular}

* Significant values, "df: Degree of freedom, F: Fishers's function, P: Level of significance, C.V: Coefficient of variation" 

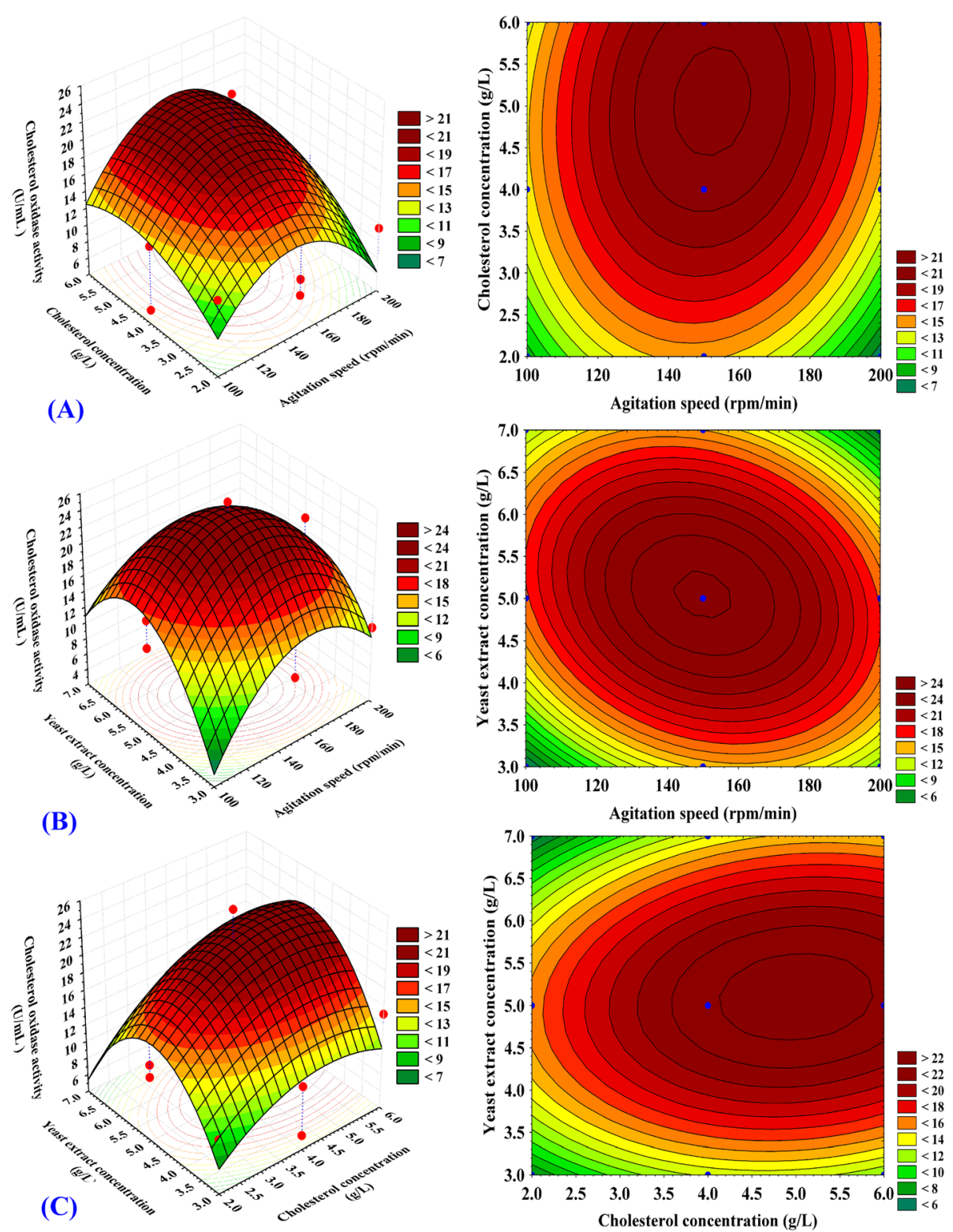

Fig. 6 a-c Three-dimensional response surface plots showing the effect of agitation speed, cholesterol, yeast extract concentration and their mutual effects on the cholesterol oxidase production

third variable is fixed at its zero level (shown in Fig. 6ac). Figure 6a represents the cholesterol oxidase production as the simultaneous effect of agitation speed $\left(\mathrm{X}_{1}\right)$, cholesterol concentration $\left(\mathrm{X}_{2}\right)$ while yeast extract was kept at the central point $(5 \mathrm{~g} / \mathrm{L})$. The cholesterol oxidase activity increases gradually by increasing cholesterol concentration and agitation speed till reach its optimum, but further increase in both cholesterol concentration and agitation speed leads to decrease in cholesterol oxidase activity. By solving the Eq. (2), the highest cholesterol oxidase production of $27.21 \mathrm{U} / \mathrm{mL}$ could be reached using $5 \mathrm{~g} / \mathrm{L}$ yeast extract at the optimal predicted levels of agitation speed and cholesterol concentration of $150 \mathrm{rpm}$ and $4.8 \mathrm{~g} / \mathrm{L}$; respectively.

The three-dimensional surface and contour plots in Fig. 6b illustrates cholesterol oxidase production as a function of agitation speed $\left(\mathrm{X}_{1}\right)$ and yeast extract concentration $\left(\mathrm{X}_{3}\right)$ while cholesterol concentration $\left(\mathrm{X}_{2}\right)$ was fixed at the central point $(4 \mathrm{~g} / \mathrm{L})$. Figure $6 \mathrm{~b}$ indicates that low agitation speed $\left(\mathrm{X}_{1}\right)$ results in lower cholesterol oxidase production and with increasing the agitation speed, the cholesterol oxidase production increases beyond 150 rpm after which cholesterol oxidase production was reduced. Lower and higher concentrations of yeast extract 
$\left(\mathrm{X}_{3}\right)$ results in lower cholesterol oxidase production, and the maximum cholesterol oxidase production, obviously obtained at the central level of the yeast extract concentration. By analysis of Fig. $6 \mathrm{~b}$ and solving the Eq. (2), the maximum predicted cholesterol oxidase production of $26.55 \mathrm{U} / \mathrm{mL}$ could be reached using $4 \mathrm{~g} / \mathrm{L}$ cholesterol at the optimal predicted levels of agitation speed $(150 \mathrm{rpm})$ and yeast extract concentration $(5 \mathrm{~g} / \mathrm{L})$.

Figure 6c shows cholesterol oxidase production as influenced by cholesterol concentration $\left(\mathrm{X}_{2}\right)$ and the concentration of yeast extract $\left(\mathrm{X}_{3}\right)$ by maintaining the agitation speed at the central point $(150 \mathrm{rpm})$. With an increased concentrations of both cholesterol and yeast extract, cholesterol oxidase production by the selected strain (Streptomyces anulatus strain NEAE-94) was improved and the maximum cholesterol oxidase production was obtained at the middle levels of two factors, and further increase of cholesterol concentration or yeast extract concentration decreases cholesterol oxidase activity. By analysis of Fig. $6 \mathrm{c}$ and solving the Eq. (2), the maximal predicted cholesterol oxidase production of $27.21 \mathrm{U} / \mathrm{mL}$ could be reached using agitation speed $(150$ rpm) and the optimal predicted levels of $4.8 \mathrm{~g} / \mathrm{L}$ cholesterol and yeast extract concentration $(5 \mathrm{~g} / \mathrm{L})$.

\section{Verification of the model}

Using the optimal levels of the process variables as obtained using Box-Behnken design, the experimental cholesterol oxidase production was verified and compared with the predicted value of cholesterol oxidase production $(26.55 \mathrm{U} / \mathrm{mL})$. The maximum experimental cholesterol oxidase production by Streptomyces anulatus strain NEAE-94 was $27.31 \mathrm{U} / \mathrm{mL}$. The verification revealed a high degree of model accuracy (97.21\%).

\section{Discussion}

\section{Taxonomic conclusions}

Streptomyces sp. strain NEAE-94 mainly have the same characteristics as Streptomyces anulatus, Streptomyces flavofuscus, Streptomyces parvus and Streptomyces fimicarius in that it produced rectiflexibiles spore chains and did not produce melanin pigments. However, Streptomyces sp. strain NEAE-94 mainly differed from Streptomyces fimicarius in that it produces yellow aerial mycelium, yellow substrate mycelium, and yellow diffusible pigment on ISP medium 2. On the other hand, Streptomyces fimicarius lost the ability to produce a diffusible pigment and not produce distinctive substrate mycelium pigment. Also Streptomyces sp. strain NEAE-94 differed from Streptomyces fimicarius and Streptomyces parvus in the pattern of utilization of carbon sources (Table 1). Moreover, Streptomyces sp. strain NEAE-94 mainly differed from Streptomyces flavofuscus in the diffusible pigment and color of substrate mycelium. The phylogenetic tree
(Fig. 4) was constructed by using the tree-making neighbor-joining algorithm method of Saitou and Nei [21] using MEGA 3.0 software [22]. The comparative study between the related species of the genus Streptomyces and Streptomyces sp. strain NEAE-94. (Table 1) indicated that it mostly related to Streptomyces anulatus [20]. Accordingly, Streptomyces sp. strain NEAE-94 was identified as Streptomyces anulatus strain NEAE-94 (accession number is KC354803).

\section{Screening of significant factors for production of cholesterol oxidase using Plackett-Burman design}

The values of the determination coefficient $\left(R^{2}=0.9996\right)$ and the adjusted determination coefficient (Adj. $R^{2}=$ 0.9978 ) are very high and suggests a strong model significance [23]. The smaller $P$-value of the factor reveals that the factor is more essential for cholesterol oxidase production.

The residuals' normal probability plot is a valuable tool for detecting and explaining the systemic deviations from the normality [24]. Figure $5 c$ displays a normal probability plot of the residuals. The residuals have been drawn against a theoretical normal model distribution in such a manner that the points for cholesterol oxidase production should form an approximately straight line. Departures from this straight line show deviations from normality. The normal probability plot of the residuals shows points close a diagonal line; so that residuals seem to be distributed nearly normal. This means the model was well designed to the findings of the experiments. Figure $5 \mathrm{~d}$ shows the plot of the predicted cholesterol oxidase production versus actual values, while the dots collected around a diagonal line reveals the model's excellent fit.

\section{Optimization of the selected significant variables by Box- Behnken design}

Depending on the statistical analysis of Plackett-Burman design results, the factors with positive impact on production of cholesterol oxidase (temperature, agitation speed, $\mathrm{pH}$, starch, cholesterol, peptone, yeast extract, ammonium sulphate and $\left.\mathrm{K}_{2} \mathrm{HPO}_{4}\right)$ have been maintained at their highest levels $(+1)$. Whereas, the factors with negative impact on cholesterol oxidase production (incubation time, inoculum size, $\mathrm{MgSO}_{4}, \mathrm{NaCl}$ and $\left.\mathrm{FeSO}_{4}\right)$ were fixed at their low $(-1)$ levels. On the other hand, the insignificant factors (starch, peptone and $\left.\left(\mathrm{NH}_{4}\right)_{2} \mathrm{SO}_{4}\right)$ have been omitted in the subsequent experiments.

The maximum cholesterol oxidase production obtained from this study $(27.31 \mathrm{U} / \mathrm{mL})$ by Streptomyces anulatus strain NEAE-94 is superior and greater than most of the other reported values such as the cholesterol oxidase production by Rhodococcus equi no. 23 ( $0.24 \mathrm{U} /$ 
mL) [25], Micrococcus sp. (3.68 U/mL) [26], Streptomyces A $(2.44 \mathrm{U} / \mathrm{mL})$ [27], Brevibacterium sp. $(1.483 \mathrm{U} / \mathrm{mL})$ [28], Streptomyces lavendulae (2.21 U/mL) [29], Streptomyces sp. (6.2 U/mL) [30], Bacillus cereus $(1.67 \mathrm{U} / \mathrm{mL})$ [31] and Streptomyces fradiae $(0.03 \mathrm{U} / \mathrm{mL})$ [32].

The current $R^{2}$ and adjusted $R^{2}$ values were 0.9951 and 0.9864; respectively, implying that the model is appropriate to represent the real relationship between cholesterol oxidase production and the selected factors. The largest $R^{2}$ value showed that experimental and expected cholesterol oxidase production values are in excellent agreement [33]. Predicted $R^{2}$ value of 0.9427 shows that the model is sufficient to predict the value of the production of cholesterol oxidase in the range of factors used.

The non- significant lack-of-fit, the high value of adjusted and predicted R-squared, low PRESS value, high $F$-value, low standard deviation and high adequate precision indicating the validity and high degree of accuracy of the model prediction for the production of cholesterol oxidase by Streptomyces anulatus strain NEAE-94.

Enhanced production of cholesterol oxidase was recorded with the use of different compounds as cholesterol, yeast extract [32]; malt extract, potato starch and peptone [34] as substrates. Yehia et al. [35] noted that the growth and breakdown of cholesterol by the tested bacterial isolates were largely and severely affected by cholesterol concentration in the culture medium. The largest cholesterol breakdown by the Enterococcus hirae was achieved using $1 \mathrm{~g} / \mathrm{L}$ cholesterol. Sojo et al. [36] and Yazdi et al. [32] reported that the largest cholesterol breakdown and maximum cholesterol oxidase production by the Rhodococcus erythropolis and Streptomyces fradiae was achieved using $2 \mathrm{~g} / \mathrm{L}$ cholesterol.

There are different and opposite effects of nitrogen sources on the production of cholesterol oxidase in the literature. Voelker and Altaba [37] recorded an increase in the production of cholesterol oxidase by organic nitrogen much greater than inorganic nitrogen. The reason is that organic nitrogen may contain the most types of growth factors and amino acids required for the microbial growth and could be immediately metabolized by cells, thereby supporting the cholesterol oxidase production [29]. Maximum cholesterol oxidase production by Rhodococcus equi $2 \mathrm{C}$ and Rhodococcus equi no. 23 was recorded using $0.3 \%$ [32] and $0.4-0.5 \%, \mathrm{w} / \mathrm{v}$ yeast extract [25]; respectively. On the other hand, Moradpour et al. [38] and Ahmad [39] reported that the best sources of nitrogen for cholesterol decomposition by Pseudonocardia compacta S-39 were ammonium nitrate, ammonium sulphate and sodium nitrate [40]. Liu et al. [41] reported that the best sources of nitrogen for maximum cholesterol oxidase production by Arthrobacter simplex were ammonium salts.
Appropriate oxygen must be given in the fermentation media using shaken cultures to satisfy the organism's growing demands and to produce the desired end product. The suitable agitation speed ensures that the dissolved oxygen in the medium is adequately supplied and can become vital for microbial biosynthesis of certain end products.

In this study, the maximum cholesterol oxidase production $(27.31 \mathrm{U} / \mathrm{mL})$ is obtained using the following medium formula $(\mathrm{g} / \mathrm{L})$ : yeast extract 5 , cholesterol 4 , $\mathrm{FeSO}_{4} .7 \mathrm{H}_{2} \mathrm{O} 0.01, \mathrm{MgSO}_{4} .7 \mathrm{H}_{2} \mathrm{O} 0.5, \mathrm{NaCl} 0.5, \mathrm{~K}_{2} \mathrm{HPO}_{4}$ $1, \mathrm{pH} 7$, inoculum size $4 \%(\mathrm{v} / \mathrm{v})$, temperature $37^{\circ} \mathrm{C}$, agitation speed $150 \mathrm{rpm}$, medium volume $50 \mathrm{~mL}$ and incubation time 5 days.

El-Naggar et al. [14] used the 2-level Plackett-Burman experimental design in 20 experimental run to evaluate the importance of 15 process variables of medium components and operating conditions for the production of cholesterol oxidase by Streptomyces cavourensis strain NEAE-42. The most important factors that significantly influenced cholesterol oxidase production were initial $\mathrm{pH}$, cholesterol and $\left(\mathrm{NH}_{4}\right)_{2} \mathrm{SO}_{4}$ concentrations. ElNaggar et al. [14] reported that the optimal levels of the three selected process factors for maximum cholesterol oxidase production $(20.521 \mathrm{U} / \mathrm{mL})$ as obtained from the central composite design were $\mathrm{pH} 8$; cholesterol concentration $3 \mathrm{~g} / \mathrm{L} ;\left(\mathrm{NH}_{4}\right)_{2} \mathrm{SO}_{4} 8 \mathrm{~g} / \mathrm{L}$. However, Ahmad and Goswami [42] optimized the medium for production of cholesterol oxidase by Rhodococcus sp. NCIM 2891 using the classical and statistical methods. They reported that the maximum cholesterol oxidase production (3.25 $\mathrm{U} / \mathrm{mL}$ ) was obtained in the statistically optimized medium under the optimal levels of the process factors that were $2.5 \mathrm{~g} / \mathrm{L}\left(\mathrm{NH}_{4}\right)_{2} \mathrm{HPO}_{4}, 9 \mathrm{~g} / \mathrm{L}$ yeast extract and $3.5 \mathrm{~g} / \mathrm{L}$ cholesterol in approximately $60 \mathrm{~h}$ of cultivation at $30^{\circ} \mathrm{C}$ and $\mathrm{pH}$ 7.0. On the other hand, Srivastava et al. [43] applied various statistical optimization techniques to enhance the cholesterol oxidase production by Streptomyces rimosus MTCC 10792. They reported that, out of the examined factors, yeast extract, dextrose, starch and ammonium carbonate were the most significant factors and the maximum cholesterol oxidase production was $5.41 \mathrm{U} / \mathrm{mL}$ in the optimized medium using the optimum concentrations of the four variables that were (g/100 mL medium): 0.05 ammonium carbonate, 0.1 starch, 0.8 dextrose and 0.99 yeast extract.

Moradpour et al. [38] screen various process variables that had a major impact on cholesterol oxidase production by Streptomyces badius using Plackett-Burman design and optimized these variables using Box-Behnken design. They reported that, yeast extract, $\mathrm{pH}$, Tween 20 and temperature were the most significant factors and the maximum cholesterol oxidase production by Streptomyces badius was $2.05 \mathrm{U} / \mathrm{mL}$ in the optimized medium 
using the optimum levels of the four variables that were determined to be: yeast extract, $0.45 \% ; \mathrm{pH}, 6.5$; Tween 20, $0.05 \%$ and $30^{\circ} \mathrm{C}$. Moreover, ElBaz et al. [44] used a two-step statistical approach to optimize the production of cholesterol oxidase from Bacillus pumilus. The maximum cholesterol oxidase production $(90 \mathrm{U} / \mathrm{mL})$ was obtained after 6 days of fermentation at $\mathrm{pH} 8$ with medium/flask ratio of 0.35 and the concentrations of cholesterol, $\mathrm{NH}_{4} \mathrm{NO}_{3}$, yeast extract and Tween 80 were $0.2,0.3,1$ and $0.2 \%$; respectively.

Kuppusamy and Kumar [31] used traditional one variable at a time method to find the key nutritional components such as different carbon sources, nitrogen sources, metal ions and different physical parameters like incubation time, temperature and $\mathrm{pH}$ to enhance cholesterol oxidase production by Bacillus cereus strain KAVK4. The highest production of cholesterol oxidase was achieved under flask conditions using the optimal levels of fructose (as a carbon source) in the production medium at a concentration of $2 \%$, ammonium nitrate (as nitrogen source) at a concentration of $0.2 \%$ and magnesium sulphate as metal ion source at a concentration of $0.03 \%$. The maximum production of cholesterol oxidase by Bacillus cereus strain KAVK4 of $1.67 \mathrm{U} / \mathrm{mL}$ was achieved at the optimum process variable values (incubation time was at $32 \mathrm{~h}, \mathrm{pH} 7.5$ at room temperature).

Yang and Zhang [28] used a three level central composite design to investigate the correlation between three independent variables (cholesterol, Tween-80 and treatment time) and cholesterol oxidase production by Brevibacterium sp. They found that, the optimal values of the three independent variables resulted in highest cholesterol oxidase production by Brevibacterium sp. (1.483 U/ $\mathrm{mL}$ ) were determined to be: $22.361(\mathrm{~min})$ treatment time, $0.2932 \%$ (v/v) Tween-80, $4.076 \mathrm{~g} / \mathrm{L}$ cholesterol. In addition, El-Naggar et al. [45] used the Plackett-Burman design to evaluate the influence of nutritional and environmental variables for cholesterol oxidase production by Streptomyces aegyptia strain NEAE-102. They found that, out of 15 variables screened by Plackett-Burman design experiments, $\mathrm{pH}$, incubation time and cholesterol concentration were the most significant variables for cholesterol oxidase production. El-Naggar et al. [45] used a face centered central composite design to optimize the levels and analyze the combined effects of $\mathrm{pH}$, incubation time and cholesterol concentration. The optimum levels of these variables for the high cholesterol oxidase production $(15.631 \mathrm{U} / \mathrm{mL})$ were determined to be: $\mathrm{pH} 6,5$ days of incubation time and $3 \mathrm{~g} / \mathrm{L}$ cholesterol.

Varma and Nene [34] studied cholesterol oxidase production by Streptomyces lavendulae NCIM 2421. A peak of cholesterol oxidase activity of $1.8 \mathrm{U} / \mathrm{mL}$ was detected at $72 \mathrm{~h}$. They found that, Streptomyces lavendulae NCIM
2421 is a constitutive producer of cholesterol oxidase where the addition of cholesterol to the medium did not enhance cholesterol oxidase activity. Whereas, Chauhan et al. [29] used orthogonal array method and response surface methodology to optimize medium for cholesterol oxidase production by Streptomyces lavendulae NCIM 2499. They reported that the model predicted maximum cholesterol oxidase production $(2.21 \mathrm{U} / \mathrm{mL})$ could be achieved after $72 \mathrm{~h}$ of incubation using the medium of the following composition $(\mathrm{g} / \mathrm{L})$ : sodium chloride 0.7 , $\mathrm{MgSO}_{4} 2, \mathrm{~K}_{2} \mathrm{HPO}_{4}$ 0.6, soyabean meal 20, malt extract 20 , and glycerol $10 \mathrm{~mL} / \mathrm{L}$. An initial $\mathrm{pH}$ of 7.5 supported the maximum production of cholesterol oxidase. Moreover, Srivastava et al. [43] standardized the process of cholesterol oxidase production by studying a different range of various parameters at shake flask level. They found that the maximum cholesterol oxidase production by Streptomyces rimosus was achieved using the optimal levels of process variables which found to be inoculum size $(3 \%, v / v), p H(7)$, incubation temperature $\left(30^{\circ} \mathrm{C}\right)$, incubation time $(48 \mathrm{~h})$ and agitation speed $(200 \mathrm{rpm})$. Fazaeli et al. [46] achieved maximum cholesterol oxidase production by Escherichia coli when the induced culture production medium was incubated for $24 \mathrm{~h}$ at $15^{\circ} \mathrm{C}$. Also, Niwas et al. [30] studied the effect of process variables on cholesterol oxidase production by Streptomyces sp. at shake flask level. The results indicated that the maximum cholesterol oxidase production $(6.2 \mathrm{U} / \mathrm{mL})$ was achieved using $0.05 \%$, w/v cholesterol, $\mathrm{pH} 7$ and $35^{\circ} \mathrm{C}, 200 \mathrm{rpm}$.

\section{Conclusion}

Streptomyces anulatus strain NEAE-94 could be used as a promising, efficient source for cholesterol oxidase production. The maximum cholesterol oxidase production after statistical optimization of fermentation process variables was $27.31 \mathrm{U} / \mathrm{mL}$ with a fold of increase $6.06 \mathrm{com}$ pared with the cholesterol oxidase production before applying Plackett-Burman design $(4.51 \mathrm{U} / \mathrm{mL})$.

\section{Methods}

\section{Microorganisms and cultural conditions}

Streptomyces sp. strain NEAE-94 was isolated from a soil sample collected from Baltim, Kafr el-Sheikh Governorate, in the north of Egypt. Streptomyces sp. strain NEAE-94 isolation has been made on the plates of starch-nitrate agar medium of the following constituents $(\mathrm{g} / \mathrm{L})$ : FeS$\mathrm{O}_{4} .7 \mathrm{H}_{2} \mathrm{O} 0.01 ; \mathrm{CaCO}_{3} 3 ; \mathrm{NaCl} 0.5 ; \mathrm{MgSO}_{4} .7 \mathrm{H}_{2} \mathrm{O} 0.5$; $\mathrm{K}_{2} \mathrm{HPO}_{4} 1 ; \mathrm{KNO}_{3} 2$; starch 20 and agar 20 in $1 \mathrm{~L}$ of the distilled water. The plates were incubated for 7 days at $30^{\circ} \mathrm{C}$. For long time preservation, the isolate was stored as spore suspensions in $20 \%$ (v/v) glycerol. Streptomyces anulatus NEAE-94 is an antagonistic actinomycete exhibited a broad antimicrobial spectrum against several bacterial 
strains including multidrug-resistant Staphylococcus aureus, E. coli, Bacillus subtilis and Pseudomonas aeruginosa [19].

\section{Streptomyces sp. strain NEAE-94 efficiency for cholesterol oxidase production using colony staining method} The efficiency of Streptomyces sp. strain NEAE-94 for the production of cholesterol oxidase was investigated on agar plate medium containing cholesterol as the sole source of carbon according to the method of ElNaggar et al. [14]. The medium contains g/L: Cholesterol 2, potassium nitrate 2 , potassium phosphate dibasic 1 , magnesium sulfate heptahydrate 0.5 , sodium chloride 0.5 , calcium carbonate 3 , ferrous sulfate heptahydrate 0.01 , agar 20 and distilled water $1 \mathrm{~L} ; \mathrm{pH}$ was adjusted to 7-7.2. A colony staining procedure was applied on the growing colonies to verify cholesterol oxidase production potential. Discs of filter papers were immersed in $100 \mathrm{mM}$ potassium buffer phosphate $(\mathrm{pH} 7.0)$ containing $0.5 \%$ cholesterol; $6 \%$ phenol; $1.7 \%$ 4-aminoantipyrine and $3 \mathrm{U} / \mathrm{mL}$ horseradish peroxidase. After that, the soaked discs were then placed on the grown colonies and incubated again for $24 \mathrm{~h}$ at room temperature. Cholesterol oxidase activity was confirmed by red color development due to the formation of quinoneimine dye [47].

Inoculum preparation and cholesterol oxidase production Two hundred fifty millilitre Erlenmeyer flasks containing $50 \mathrm{~mL}$ of broth medium, comprised of g/L: $\left(\mathrm{NH}_{4}\right)_{2} \mathrm{SO}_{4}$ 7.5; cholesterol 2; $\mathrm{K}_{2} \mathrm{HPO}_{4} 1 ; \mathrm{MgSO}_{4} .7 \mathrm{H}_{2} \mathrm{O}$ 0.5; FeS$\mathrm{O}_{4} .7 \mathrm{H}_{2} \mathrm{O} \quad 0.02 ; \mathrm{ZnSO}_{4} \quad 0.002 ; \mathrm{CaSO}_{4} \quad 0.002 ; \mathrm{MnSO}_{4}$ 0.008; $\mathrm{NaCl} 1 ; \mathrm{CaCl}_{2}$ 0.0002; peptone 4; yeast extract 6; starch 9; glucose 12; Tween 80 0.05) [32] were inoculated with five disks $(9 \mathrm{~mm}$ diameter) taken from the stock culture of Streptomyces sp. strain NEAE-94 grown on starch nitrate agar medium. The inoculated flasks were incubated at $30^{\circ} \mathrm{C}$ for $48 \mathrm{~h}$ in rotating shaker incubator at $200 \mathrm{rpm}$, and used as inoculum for subsequent experiments.

Fifty millilitre of the fermentation medium was inoculated with the prepared inoculum and incubated in rotating shaker incubator at $30-37^{\circ} \mathrm{C}$ and $150-200 \mathrm{rpm}$. After the incubation time, the cell free- culture supernatant containing the crude enzyme was obtained by centrifugation of the mycelium culture using refrigerated centrifuge at $4^{\circ} \mathrm{C}$ and $6000 \times g$ for $15 \mathrm{~min}$.

\section{Assessment of cholesterol oxidase activity}

The cholesterol oxidase activity was determined spectrophotometry by the method of El-Naggar et al. [45]. One enzyme activity unit $(\mathrm{U})$ : is the quantity of the enzyme needed for forming one $\mu \mathrm{mol} \mathrm{H}_{2} \mathrm{O}_{2} / \mathrm{min}$ at $37^{\circ} \mathrm{C}$.

\section{Cultural and morphological properties of Streptomyces sp.} strain NEAE-94

Spore mass color, substrate mycelium color (reverse side of the colony) and soluble diffusible pigments were investigated on ISP media as described by the method of Shirling and Gottlieb [48] method. The spore chain morphology was examined on starch-nitrate agar medium with Scanning Electron Microscopy "Jeol JSM6360 LA operates at $20 \mathrm{Kv}$.

\section{Physiological properties}

The ability of the strain to utilize carbon sources was determined according to the method described by Shirling and Gottlieb [48] using 13 sterilized carbon sources, namely: sucrose, ribose, D (+) glucose, L-arabinose, D $(+)$ xylose, D (+) galactose, D (-) fructose, D (+) mannose, maltose, raffinose, rhamnose, trehalose and cellulose. Decomposition of cellulose was determined using Hutchinson liquid medium [49], cellulose was used in the final concentration of $1 \%, \mathrm{~W} / \mathrm{V}$, and the medium was inoculated with the spore suspension of the strain. The strain ability to reduce nitrate to nitrite was determined using Giltay liquid medium [50]. The degradation of lecithin "lecithinase activity" was determined on plates of egg-yolk medium using Nitsch and Kützner [51] method. Starch hydrolysis (amylase activity) by the strain was determined using starch-nitrate agar plates. Spore suspension was inoculated by streak the organism on the plates and incubated for 7 days at $30^{\circ} \mathrm{C}$. After that, the plates were flooded with the solution of iodine. The presence of clear zone around the growth indicated positive amylase activity [52]. Test tubes containing skimmed milk were sterilized, inoculated with spore suspension and the degree of coagulation and peptonization of milk were recorded after 7 days of incubation at $30^{\circ} \mathrm{C}$ [53]. Liquefaction of gelatin as a proteolytic character was determined following the method described by Preobrazhenskaya [54]. Production of melanin by Streptomyces sp. strain NEAE-94 was determined on slants of ISP media 1, 6 and 7; the production of brown or black soluble pigments was recorded after 7 days of incubation at $30^{\circ} \mathrm{C}$ that indicates positive production of melanin [48]. Degradation of casein [55], L-asparaginase production [56], sodium chloride tolerance [57], chitosanase [58] and uricase activities [59] were tested. The production of antimicrobial agents by Streptomyces sp. strain NEAE-94 was investigated on agar plate medium according to the method of El-Naggar et al. [19].

\section{Molecular characterization}

According to the method described by Sambrook et al. [60], the total genomic DNA of the strain was prepared. The PCR reaction was carried out using El-Naggar et al. [61] method. The amplification of $16 \mathrm{~S}$ rRNA gene from 
the Streptomyces sp. under investigation was carried out via polymerase chain reaction (PCR) using universal primer designed to amplify the full length of the gene. The reverse primer was 1492r (5'-TACGGYTACCTTGT TACGACTT-3') and the forward primer was $27 f\left(5^{\prime}\right.$ AGAGTTTGATCMTGCCTCAG-3'). The PCR reaction was conducted in a total volume of $100 \mu \mathrm{L}$ that contains $0.5 \mu \mathrm{L}$ Taq polymerase, $10 \mu \mathrm{L}$ deoxyribonucleotide $5^{\prime}$ triphosphate (dNTP's) $(250 \mathrm{mM}), 4 \mu \mathrm{L}$ of both forward and reverse primers of $10 \mathrm{pmol}, 1 \mu \mathrm{L}$ template DNA 50 ng, $3.5 \mu \mathrm{L} \mathrm{MgCl}_{2}(25 \mathrm{Mm}), 10 \mu \mathrm{L}$ PCR buffer, up to $100 \mu \mathrm{L}$ with water.

The amplified DNA was sequenced and the resulting sequence of 16S rRNA was deposited under accession number KC354803 in the GenBank NCBI database. 16S rRNA gene sequence (1536 bp) of Streptomyces sp. strain NEAE-94 was aligned with the 16S rRNA sequences of the related species of Streptomyces by using BLAST program [62]. The phylogenetic tree was built using version 2.1of MEGA4 software [22] via the neighbor-joining algorithm method.

\section{Selection of significant variables for cholesterol oxidase production by Plackett-Burman design}

The Plackett-Burman statistical design [63] is an efficient method used to screen and to identify the significant variables among large number of variables that have significant effects on a process $[64,65]$. PlackettBurman design was used in this study to select the medium components and environmental conditions that had a significant effect, either negatively or positively on cholesterol oxidase production out of 15 independent variables. The Plackett-Burman experiment was conducted in 20 runs to study the effect of the selected 15 variables on cholesterol oxidase production based on their main effects. Each variable was examined in low $(-)$ and high $(+)$ levels. The chosen factors were glucose (5 and $10 \mathrm{~g} / \mathrm{L}$ ), starch (5 and $10 \mathrm{~g} / \mathrm{L}$ ), cholesterol (1 and $3 \mathrm{~g} / \mathrm{L}$ ), yeast extract ( 1 and $4 \mathrm{~g} / \mathrm{L})$, peptone $(1$ and $4 \mathrm{~g} / \mathrm{L})$, $\left(\mathrm{NH}_{4}\right)_{2} \mathrm{SO}_{4}(4$ and $8 \mathrm{~g} / \mathrm{L}), \mathrm{FeSO}_{4} .7 \mathrm{H}_{2} \mathrm{O}(0.01$ and $0.05 \mathrm{~g} /$ $\mathrm{L}), \mathrm{MgSO}_{4} .7 \mathrm{H}_{2} \mathrm{O}(0.1$ and $0.5 \mathrm{~g} / \mathrm{L}), \mathrm{NaCl}(0.5$ and $1 \mathrm{~g} / \mathrm{L})$, $\mathrm{K}_{2} \mathrm{HPO}_{4}(0.5$ and $1 \mathrm{~g} / \mathrm{L}), \mathrm{pH}$ (7 and 9), inoculum size (2 and $4 \%, \mathrm{v} / \mathrm{v})$, incubation time (5 and 7 days), temperature $\left(30\right.$ and $\left.37^{\circ} \mathrm{C}\right)$ and agitation speed $(100$ and $150 \mathrm{rpm})$. As well, 4 dummy variables $\left(D_{1}-D_{4}\right)$ were included.

Plackett-Burman design is based on the first order model:

$$
Y=\beta_{0}+\sum \beta_{i} X_{i}
$$

Where, $\mathrm{Y}$ is cholesterol oxidase production, $\beta_{0}$ is the model intercept, $X_{\mathrm{i}}$ is the level of each independent variable and $\beta_{i}$ is the linear coefficient.

\section{Optimization of cholesterol oxidase production by Box- Behnken design (BBD)}

Based on the results of Plackett-Burman experiments, three factors with the highest $P$-values, $F$-values, effects and contribution \% were selected for further optimization using Box-Behnken design [66]. These factors were agitation speed $\left(\mathrm{X}_{1}, 100-200 \mathrm{rpm}\right)$, cholesterol concentration $\left(\mathrm{X}_{2}, 2-6 \mathrm{~g} / \mathrm{L}\right)$ and yeast extract concentration $\left(\mathrm{X}_{3}, 3-7 \mathrm{~g} / \mathrm{L}\right)$. Each factor varies on three different levels $(-1,0,+1)$, with three center points resulting in a total of 15 different experiments. BBD was used to estimate the optimal levels of these variables and to study the individual and interaction effects between the selected process variables affecting cholesterol oxidase production. Linear, quadratic and interaction effects of the three significant independent variables on cholesterol oxidase production were calculated to correlate the relationship between production of cholesterol oxidase $(\mathrm{Y})$ and these factors and to predict their optimal levels using the following second order polynomial equation:

$$
Y=\beta_{0}+\sum_{i} \beta_{i} X_{i}+\sum_{i i} \beta_{i i} X_{i}^{2}+\sum_{i j} \beta_{i j} X_{i} X_{j}
$$

In which " $\mathrm{Y}$ is the cholesterol oxidase production, $\mathrm{X}_{\mathrm{i}}$ is the coded levels of independent variables, $\beta_{0}$ is the regression coefficients, $\beta_{\mathrm{ij}}$ is the interaction coefficients, $\beta_{\mathrm{i}}$ is the linear coefficient and $\beta_{\mathrm{ii}}$ is the quadratic coefficients".

\section{Statistical analysis}

"The experimental designs and statistical analysis were performed using Design Expert software version 7 for Windows. The 3D surface plots were drawn using STATISTICA software version 8."

\section{Supplementary information}

Supplementary information accompanies this paper at https://doi.org/10. 1186/s12866-020-01775-x.

\section{Additional file 1.}

\section{Abbreviations}

rRNA: Ribosomal ribonucleic acid; FAD: Flavin adenine dinucleotide; HIV: Human immunodeficiency virus; RSM: Response surface methodology; $P$ : Level of significance; $R^{2}$ : Determination coefficient; Adj. $R^{2}$ : Adjusted determination coefficient; F: Fisher's function; ANOVA: Analysis of variance; CV: Coefficient of variation; PRESS: Predicted residual sum of squares; DNA: Deoxyribonucleic acid; PCR: Polymerase chain reaction; dNTP'S: Deoxyribonucleotide 5'- triphosphate; BBD: Box- Behnken design

\section{Acknowledgements \\ Not applicable.}

Authors' contributions

NEE proposed the research concept, designed the experiments, providing necessary tools for experiments, experimental instructions, performed the statistical analysis, analyzed and interpreted the data and wrote the 
manuscript. NME carried out the experiments, participated in writing of the manuscript. All authors read and approved the final manuscript.

\section{Funding}

Not applicable.

\section{Availability of data and materials}

All data generated or analyzed during this study are included in this published article [and its supplementary information files].

\section{Ethics approval and consent to participate}

Not applicable.

\section{Consent for publication}

Not applicable.

\section{Competing interests}

The authors declare that they have no competing interests.

Received: 16 October 2019 Accepted: 30 March 2020 Published online: 10 April 2020

\section{References}

1. Yamada K, Koroleva A, Laughlin M, Oksanen N, Akhgari A, Safronova V, Yakovleva E, Kolodyaznaya V, Buldakova T, Mikko Metsä K. Characterization and overproduction of cell-associated cholesterol oxidase ChoD from Streptomyces lavendulae YAKB-15. Sci Rep. 2019;9:11850.

2. Khan R, Solanki P, Kaushik A, Singh S, Ahmad S, Malhotra BD. Cholesterol biosensor based on electrochemically prepared polyaniline conducting polymer film in presence of a nonionic surfactant. J Polym Res. 2009:16:363-73.

3. Molaei R, Sabzi RE, Farhadi K, Kheiri F, Forough M. Amperometric biosenso for cholesterol based on novel nanocomposite array gold nanoparticles/ acetone-extracted propolis/multiwall carbon nanotubes/gold. Micro Nano Lett. 2014;9:100-4

4. National Institutes of Health. Third report of the expert panel on detection, evaluation, and treatment of high blood cholesterol in adults. Bethesda: $\mathrm{NIH}$ Publication 1, National Institutes of Health; 2001. p. 3670.

5. Kumari L, Kanwar SS. Cholesterol oxidase and its applications. Adv Microbiol. 2012;2:49-65.

6. Mendes MV, Recio E, Anton N, Guerra SM, Santos Aberturas J, Martín JF, Aparicio JF. Cholesterol oxidases act as signaling proteins for the biosynthesis of the polyene macrolide pimaricin. Chem Biol. 2007;14:279-90.

7. Pollegioni L, Piubelli L, Molla G. Cholesterol oxidase. Biotechnol J. 2009;276: 6857-70.

8. Liu J, Xian G, Li M, Zhang Y, Yang M, Yu Y, Gao L. Cholesterol oxidase from Bordetella sp. promotes irreversible cell apoptosis in lung adenocarcinoma by cholesterol oxidation. Cell Death and Dis. 2014;5:1372.

9. Brzostek A, Dziadek B, Rumijowska-Galewicz A, Pawelczyk J, Dziadek J. Cholesterol oxidase is required for virulence of Mycobacterium tuberculosis. FEMS Microbiol Lett. 2007;275:106-12.

10. Navas J, Gonzalez-Zorn B, Ladron N, Garrido P, Vazquez-Boland JA. Identification and mutagenesis by allelic exchange of choE, encoding a cholesterol oxidase from the intracellular pathogen Rhodococcus equi. J Bacteriol. 2001;183:4796-805

11. Purcell JP, Greenplate JT, Jennings MJ, Ryerse JS, Pershing JC, Sims SR, Stonard RJ. Cholesterol oxidase: a potent insecticidal active against boll weevil larvae. Biochem Biophys Res Commun. 1993;196:1406-13.

12. Ono A, Freed EO. Plasma membrane rafts play a critical role in HIV-1 assembly and release. Proc Natl Acad Sci U S A. 2001:98:13925-30.

13. Bavari S, Bosio CM, Wiegand E, Ruthel G, Will AB, Geisbert TW, Hevey M, Schmaljohn C, Schmaljohn A, Aman MJ. Lipid raft micro domains: a gateway for compartmentalized trafficking of ebola and Marburg viruses. J Exp Med. 2002;195:593-602.

14. El-Naggar NE, El-Shweihy NM, El-Ewasy SM. Identification and statistical optimization of fermentation conditions for a newly isolated extracellular cholesterol oxidase-producing Streptomyces cavourensis strain NEAE-42. BMC Microbiol. 2016a;16:217.

15. Fazaeli A, Golestani A, Lakzaei M, SSR V, Aminian M. Expression optimization, purification, and functional characterization of cholesterol oxidase from Chromobacterium sp. DS1. PloS one. 2019;14(2). https://doi.org/10.1371/ journal.pone.0212217.
16. Wali H, Rehman FU, Umar A, Ahmed S. Cholesterol degradation and production of extracellular cholesterol oxidase from Bacillus pumilus W1 and Serratia marcescens W8: BioMed research international; 2019: 1359528.

17. Sahu S, Shera SS, Banik RM. Optimization of process parameters for cholesterol oxidase production by Streptomyces Olivaceus MTCC 6820. Open Biotechnol J. 2019; 13(1):47-58. .

18. Chang CY, Lee CL, Pan TM. Statistical optimization of medium components for the production of Antrodia cinnamomea AC0623 in submerged cultures. Appl Microbiol Biotechnol. 2006;72:654-61.

19. El-Naggar NE, El-Bindary AA, Salah N. Statistical optimization of process variables for antimicrobial metabolites production by Streptomyces anulatus NEAE-94 against some multidrug-resistant strains. Int J Pharma. 2013;9:322-34.

20. Goodfellow M, Kämpfer P, Busse HJ, Trujillo ME, Suzuki Kl, Ludwig W, Whitman WB. Bergey's manual of systematic bacteriology, second edition, The Actinobacteria Part A, vol. 5. 2nd ed. New York Dordrecht Heidelberg London: Springer; 2012.

21. Saitou N, Nei M. The neighbor joining method: a new method for reconstructing phylogenetic trees. Mol Biol Evol. 1987;4:406-25.

22. Tamura K, Dudley J, Nei M, Kumar S. MEGA4: molecular evolutionary genetics analysis (MEGA) software version 4.0. Mol Biol Evol. 2007;24:1596-9.

23. Akhnazarova S, Kafarov V. Experiment optimization in chemistry and chemical engineering. Moscow: Mir Publishers; 1982.

24. Montgomery, D. C. Design and analysis of experiments, 3rd edn. Wiley, New York (1991). .

25. Lee MT, Chen WC, Chou CC. Maximization of cholesterol oxidase production by Rhodococcus equi no. 23 by using response surface methodology. Biotechnol Appl Biochem. 1998;28:229-33.

26. Kanchana R, Correia D, Sarkar S, Gawde P, Rodrigues A. Production and partial characterization of cholesterol oxidase from Micrococcus sp. isolated from Goa, India. Int J Appl Biol Pharma Tech. 2011:2:393-8.

27. Lashgarian HE, Jahanbakhsh S, Shahzamani K. Molecular identification of cholesterol oxidase enzyme-producing Streptomyces bacteria in soil of Lorestan province, Iran. Int J Medical Res Health Sci. 2016;5:54-62.

28. Yang S, Zhang $\mathrm{H}$. Optimization of cholesterol oxidase production by Brevibacterium sp. employing response surface methodology. Afr J Biotechnol. 2012:11:8316-22.

29. Chauhan AK, Survase SA, Kumar JK, Annapure US. Medium optimization by orthogonal array and response surface methodology for cholesterol oxidase production by Streptomyces lavendulae NCIM 2499. J Gen Appl Microbiol. 2009:55(3):171-80.

30. Niwas $R$, Singh $V$, Singh $R$, Tripathi $D$, Tripathi CKM. Production, purification and characterization of cholesterol oxidase from a newly isolated Streptomyces sp. World J Microbiol Biotechnol. 2013;29(11):2077-85.

31. Kuppusamy A, Kumar KV. Optimization of cholesterol oxidase production and 165 rRNA partial sequence of Bacillus cereus strain KAVK4 isolated from butter. J Appl Pharm Sci. 2016;6:061-6.

32. Yazdi MT, Kamranpour N, Zahraei M, Agaiepour K. Streptomyces fradiae, a potent producer of cholesterol oxidase. Iran J Sci Technol Trans. 1999;3:151-7.

33. Weisberg S. Applied linear regression. 2nd ed. New York: Wiley; 1985.

34. Varma R, Nene S. Biosynthesis of cholesterol oxidase by Streptomyces lavendulae NCIM 2421. Enzyme Microb Tech. 2003;33:286-91.

35. Yehia HM, Hassanein WA, Ibraheim SM. Purification and characterisation of the extracellular cholesterol oxidase enzyme from Enterococcus hirae. BMC Microbiol. 2015;15:178. .

36. Sojo MM, Bru RR, Garcia FF. Rhodococcus erythropolis ATCC 25544 as a suitable source of cholesterol oxidase: cell-linked and extracellular enzyme synthesis, purification and concentration. BMC Microbiol. 2002;2:3.

37. Voelker F, Altaba S. Nitrogen source governs the patterns of growth and pristinamycin production in Streptomyces pristinaespiralis. Microbiol. 2001; 147(9):2447-59.

38. Moradpour Z, Ghasemian A, Safari A, Mohkam M, Ghasemi Y. Isolation, molecular identification and statistical optimization of culture condition for a new extracellular cholesterol oxidase producing strain using response surface methodology. Ann Microbiol. 2012;63:941-50.

39. Ahmad S. Studies on production characterization and application potential of cholesterol oxidase from Rhodococcus sp. NCIM 2891; A Thesis. Assam: Indian Institute of Technology Guwahati; 2014.

40. Sabry AA. Decomposition of cholesterol by some organisms isolated from certain Egyptian soils, M.Sc. Thesis. Bot and Microbiol Department, Al-Azhar University, Cairo, 1994. 
41. Liu WH, Meng MH, Chen KS. Purifcation and some properties of cholesterol oxidases produced by an inducible and a constitutive mutant of Arthrobacter simplex. Agri Biol Chem. 1988;52:413-8.

42. Ahmad S, Goswami P. Enhanced production of cell-bound cholesterol oxidase from Rhodococcus sp. NCIM 2891 by the statistical method. Ann Microbiol. 2012:63:199-205.

43. Srivastava A, Singh V, Haque S, Pandey S, Mishra M, Jawed A, Shukla PK, Singh PK, Tripathi CKM. Response surface methodology genetic algorithm based medium optimization, purifcation, and characterization of cholesterol oxidase from Streptomyces rimosus. Sci Rep. 2018; 8:10913.

44. ElBaz FN, Gamal RF, AF EB, Ibrahim NE, ElMekawy A. Biochemical and biotechnological studies on a novel purified Bacillus cholesterol oxidase tolerant to solvent and thermal stress. Biocatalysis Biotransform. 2017;35: 205-14.

45. El-Naggar NE, Soliman HM, El-Shweihy NM. Extracellular cholesterol oxidase production by Streptomyces aegyptia, in vitro anticancer activities against rhabdomyosarcoma, breast cancer cell-lines and in vivo apoptosis. Sci Rep. 2018; 8: 2706.

46. Fazaeli A, Golestani A, Lakzaei M, Varaei SSR, Aminian M. Expression optimization of recombinant cholesterol oxidase in Escherichia coli and its purifcation and characterization. AMB Expr. 2018:8(1):183.

47. Lashkarian HE, Raheb J, Shahzamani K, Shahbani H, Shamsara M, Hajipour M. Isolation and identification of a native Rhodococcus strain producing cholesterol oxidase from soil. Int J Biotechnol Biochem. 2010;6:205-17.

48. Shirling EB, Gottlieb D. Methods for characterization of Streptomyces species. Int J Syst Bacteriol. 1966;16:313-40.

49. Crawford DL, McCoy E. Cellulases of Thermomonospora fusca and Streptomyces thermodiastaticus. Appl microbial. 1972;24:150-2.

50. Williams ST, Goodfellow M, Alderson G, Wellington EMH, Sneath PHA, Sackin MJ. Numerical classification of Streptomyces and related genera. J Gen Microbiol. 1983;129:1743-813.

51. Nitsch B, Kützner H. Egg-yolk agar as a diagnostic medium for streptomycetes. Experientia. 1969;25:220-1.

52. Mishra S, Behera N. Amylase activity of a starch degrading bacteria isolated from soil receiving kitchen wastes. Afr J Biotechnol. 2008;7:3326-31.

53. Cowan ST, Steel KJ. Cowan and Steel's manual for identification of medical bacteria. 2nd ed. London: Cambridge University Press; 1974.

54. Preobrazhenskaya T. Taxonomy problems of genus Actinomyces (Streptomyces), The actinomycetales. Jena: VEB Gustav Fischer Verlag; 1970. p. 91-6.

55. Gordon RE, Barnett DA, Handerhan JE, Pang CHN. Nocardia coeliaca, Nocardia autotrophica, and the nocardin strain. Int J Syst Bacteriol. 1974;24: 54-63.

56. El-Naggar NE. Extracellular production of the oncolytic enzyme, Lasparaginase, by newly isolated Streptomyces sp. strain NEAE-95 as potential microbial cell factories: optimization of culture conditions using response surface methodology. Curr Pharm Biotechnol. 2015a; 16(2):162-78.

57. Tresner H, Hayes JA, Backus E. Differential tolerance of streptomycetes to sodium chloride as a taxonomic aid. Appl Microbial. 1968;16:1134-6.

58. Choi YJ, Kim EJ, Piao Z, Yun YC, Shin YC. Purification and characterization of chitosanase from Bacillus sp. strain KCTC 0377BP and its application for the production of chitosan oligosaccharides. Appl Environ Microbiol. 2004;70: 4522-31.

59. El-Naggar NE. Isolation, screening and identification of actinobacteria with uricase activity: statistical optimization of fermentation conditions for improved production of uricase by Streptomyces rochei NEAE-25. Int J Pharmacol. 2015b;11:644-58

60. Sambrook J, Fritsch EF, Maniaties T. Molecular cloning: a laboratory manual. 2nd ed. Cold Spring Harbor: Cold Spring Harb Lab Press; 1989.

61. El-Naggar NE, Sherief AA, Hamza SS. Streptomyces aegyptia sp. nov., a novel cellulolytic streptomycete isolated from soil in Egypt. Afr J Microbiol Res. 2011;29:5308-15.

62. Altschul SF, Madden TL, Schäffer AA, Zhang J, Zhang Z, Miller W, Lipman DJ. Gapped BLAST and PSI-BLAST: a new generation of protein database search programs. Nucleic Acids Res. 1997;25:3389-402.

63. Plackett RL, Burnam JP. The design of optimum multifactorial experiments. Biometrika. 1946;33:305-25.

64. El-Naggar NE, Moawad H, El-Shweihy NM, El-Ewasy SM. Optimization of culture conditions for production of the anti-leukemic glutaminase free Lasparaginase by newly isolated Streptomyces olivaceus NEAE-119 using response surface methodology: BioMed research international; 2015, 2015: 627031-6270315.

65. El-Naggar NE, Hamouda RA. Antimicrobial potentialities of Streptomyces lienomycini NEAE-31 against human pathogen multidrug-resistant Pseudomonas aeruginosa. Int J Pharma. 2016b;12:769-88.

66. Box GEP, Behnken DW. Some new three level designs for the study of quantitative variable. Technometrics. 1960;2:455-63.

\section{Publisher's Note}

Springer Nature remains neutral with regard to jurisdictional claims in published maps and institutional affiliations.
Ready to submit your research? Choose BMC and benefit from:

- fast, convenient online submission

- thorough peer review by experienced researchers in your field

- rapid publication on acceptance

- support for research data, including large and complex data types

- gold Open Access which fosters wider collaboration and increased citations

- maximum visibility for your research: over $100 \mathrm{M}$ website views per year

At BMC, research is always in progress.

Learn more biomedcentral.com/submissions 